\title{
Effect of yeast species and processing on intestinal microbiota of Atlantic salmon (Salmo salar) fed soybean meal-based diets in seawater
}

Jeleel 0. Agboola ( $\square$ jeleel.opeyemi.agboola@nmbu.no)

Department of Animal and Aquacultural Sciences, Norwegian University of Life Sciences, P.0. Box 5003, NO-1432 Ås

\section{Sérgio D.C. Rocha}

Department of Animal and Aquacultural Sciences, Norwegian University of Life Sciences, P.O. Box 5003, NO-1432 Ås

\section{Dominic D. Mensah}

Department of Animal and Aquacultural Sciences, Norwegian University of Life Sciences, P.0. Box 5003, NO-1432 Ås

\section{Jon $\varnothing$. Hansen}

Department of Animal and Aquacultural Sciences, Norwegian University of Life Sciences, P.0. Box 5003, NO-1432 Ås

\section{Ove Øyås}

Department of Animal and Aquacultural Sciences, Norwegian University of Life Sciences, P.0. Box 5003, No-1432 Ås

\section{David Lapeña}

Faculty of Chemistry, Biotechnology and Food Science, Norwegian University of Life Sciences, P.O. Box 5003, NO-1432 Ås

\section{Liv T. Mydland}

Department of Animal and Aquacultural Sciences, Norwegian University of Life Sciences, P.O. Box 5003, No-1432 Ås

\section{Magnus $\emptyset$. Arntzen}

Faculty of Chemistry, Biotechnology and Food Science, Norwegian University of Life Sciences, P.O. Box 5003, NO-1432 Ås

\section{Svein J. Horn}

Faculty of Chemistry, Biotechnology and Food Science, Norwegian University of Life Sciences, P.O. Box 5003, NO-1432 Ås

\section{Margareth Øverland}

Department of Animal and Aquacultural Sciences, Norwegian University of Life Sciences, P.0. Box 5003, No-1432 Ås 


\section{Research Article}

Keywords: Cyberlindnera jadinii, Wickerhamomyces anomalus, gut microbiota, predicted metabolic capacity, SBMIE, microbial diversity, inactivated, autolysis

Posted Date: February 16th, 2022

DOI: https://doi.org/10.21203/rs.3.rs-1351266/v1

License: (c) (i) This work is licensed under a Creative Commons Attribution 4.0 International License.

Read Full License 


\title{
Effect of yeast species and processing on intestinal microbiota of Atlantic salmon (Salmo salar) fed soybean meal-based diets in seawater
}

\author{
Jeleel O. Agboola ${ }^{1}{ }^{*}$, Sérgio D.C. Rocha ${ }^{1}$, Dominic D. Mensah ${ }^{1}$, Jon Ø. Hansen ${ }^{1}$, Ove Øyås ${ }^{1,2}$, David Lapeña ${ }^{2}$, Liv T. \\ Mydland $^{1}$, Magnus Ø. Arntzen ${ }^{2}$, Svein J. Horn ${ }^{2}$, and Margareth Øverland ${ }^{1 *} \bowtie$ \\ ${ }^{1}$ Department of Animal and Aquacultural Sciences, Norwegian University of Life Sciences, P.O. Box 5003, NO-1432 Ås, Norway \\ ${ }^{2}$ Faculty of Chemistry, Biotechnology and Food Science, Norwegian University of Life Sciences, P.O. Box 5003, NO-1432 Ås, Norway
}

\begin{abstract}
Background: Yeasts are gaining attention as alternative ingredients in aquafeeds. However, the impact of yeast inclusion on modulation of intestinal microbiota of fish fed plant-based ingredients is limited. Thus, the present study investigates the effects of yeast and processing on composition, diversity and predicted metabolic capacity of gut microbiota of Atlantic salmon smolt fed soybean meal (SBM)based diet. Two yeasts, Cyberlindnera jadinii (CJ) and Wickerhamomyces anomalus (WA), were produced in-house and processed by direct heat-inactivation with spray-drying (ICJ and IWA) or autolyzed at $50{ }^{\circ} \mathrm{C}$ for $16 \mathrm{~h}$, followed by spray-drying (ACJ and AWA). In a 42-day feeding experiment, fish were fed one of six diets: a fishmeal (FM)-based diet, a challenging diet with 30\% SBM and four other diets containing $30 \%$ SBM and $10 \%$ of each of the four yeast products (i.e., ICJ, ACJ, IWA and AWA). Microbial profiling of digesta samples was conducted using 16S rRNA gene sequencing, and the predicted metabolic capacities of gut microbiota were determined using genome-scale metabolic models.

Results: The microbial composition and predicted metabolic capacity of gut microbiota differed between fish fed FM diet and those fed SBM diet. The digesta of fish fed SBM diet was dominated by members of lactic acid bacteria, which was similar to microbial composition in the digesta of fish fed the inactivated yeasts (ICJ and IWA diets). Inclusion of autolyzed yeasts (ACJ and AWA diets) reduced the richness and diversity of gut microbiota in fish. The gut microbiota of fish fed ACJ diet was dominated by the genus Pediococcus and showed a predicted increase in mucin O-glycan degradation compared with the other diets. The gut microbiota of fish fed AWA diet was highly dominated by the family Bacillaceae.

Conclusions: The present study showed that dietary inclusion of FM and SBM differentially modulate the composition and predicted metabolic capacity of gut microbiota of fish. The inclusion of inactivated yeasts did not alter the modulation caused by SBM-based diet. Fish fed ACJ diet increased relative abundance of Pediococcus, and mucin O-glycan degradation pathway compared with the other diets.
\end{abstract}

Cyberlindnera jadinii | Wickerhamomyces anomalus | gut microbiota | predicted metabolic capacity | SBMIE | microbial diversity | inactivated | autolysis

Correspondence: jeleel.opeyemi.agboola@nmbu.no

Correspondence: margareth.overland@nmbu.no

\section{${ }_{41}$ Background}

42 Plant protein sources are increasingly being used in commer43 cial aquafeeds $(1,2)$. Among the plant-based ingredients, 44 the use of soybean meal (SBM) in diets of Atlantic salmon is restricted due to the presence of anti-nutritional factors (such as trypsin inhibitors, protease inhibitors and saponin) that compromise the growth performance, nutrient digestibility, and health of fish $(3,4)$. A number of studies $(5-10)$ have reported that dietary inclusion of SBM induce inflammation in the distal intestine of Atlantic salmon; a condition widely known as SBM-induced enteritis (SBMIE), which is characterized by loss of enterocyte vacuolization, reduction in mucosal fold height, and infiltration of inflammatory cells in the lamina propria and epithelial submucosa. Considering these limitations, a refined soy-product known as soy-protein concentrate (SPC) with low level of anti-nutritional factors, is currently used in commercial salmon diets. The use of plant ingredients such as SPC in aquafeeds also raises ethical and environmental concerns as continuous use of SPC in aquafeeds may increase pressure on cultivable land, water and energy use, as well as decrease their availability for direct human consumption $(11,12)$. Therefore, there is an emerging need for sustainable novel ingredients for aquaculture.

Microbial ingredients such as yeasts are gaining attention as potential novel ingredient in aquaculture due to their ability to convert low-value by-products into high-value resources (13), high nutritional values (14-16), low environmental footprint (17) and functional effects in fish $(18,19)$. Studies have shown that dietary inclusion of yeasts could alleviate adverse effects of SBM in Atlantic salmon $(18,19)$, but little is known of their effects on intestinal microbiota of fish. The gut microbiota plays important roles in host physiological and metabolic processes, such as digestive function, growth performance, immune function, and health (20-22). A number of studies (23-26) have documented the effects of SBM inclusion on intestinal microbiota of Atlantic salmon. Identifying microbiota modulated by inclusion of yeasts in the diets may be crucial for improving nutrient utilization, growth performance, and health of Atlantic salmon fed plantbased diets. Therefore, the objective of the present study was to examine the effect of yeast species and processing on richness, diversity and predicted metabolic profile of gut microbiota of Atlantic salmon fed SBM-based diet in seawater. Two yeasts, Cyberlindnera jadinii (CJ) and Wickerhamomyces anomalus (WA) produced from wood sugars using in-house bioreactors, were used in the current study. 


\section{Results}

Characteristics of sequence data. After the sequence denoising, ASV filtering and clustering, a total number of 6.6 million reads were retained for the downstream data analysis. The median of reads per sample used for downstream analysis was 23,087, with the minimum and maximum values being 1,604 and 180,844, respectively. The reads for the downstream analysis generated a total of 906 unique ASVs, of which $76.4 \%$ were assigned at the genus level and $13.5 \%$ annotated at the species level.

\section{Microbiota composition of mock and negative con-}

trols. All the eight bacterial species expected in the mock were successfully identified at genus level, with only Staphylococcus aureus being identified at species level (Fig. S1). The relative abundance of $S$. aureus was correctly estimated, whereas the abundance of Salmonella, Pseudomonas and Escherichia-Shigella were overestimated. Contrary, the relative abundance of Listeria, Lactobacillus, Enterococcus and Bacillus were underestimated. The average Pearson correlation coefficient (Pearson's $r$ ) between the expected and the observed taxonomic profile of the mock was 0.30 , whereas the Pearson's $r$ between the observed mock was 0.99 . The dominant taxa identified as contaminants in the negative controls and the blank filter papers were Actinobacteria (47\%), Bacilli (18\%), and Gammaproteobacteria (15\%) (Table S1).

Microbiota associated with feed and water. At phylum level, the feed-associated microbiota was dominated by Firmicutes and Proteobacteria (Fig. 1A). The ACJ (89\%) and AWA (94\%) feeds had higher abundance of Firmicutes compared with the remaining feeds $(72-80 \%)$. On the other hand, the relative abundance of Proteobacteria was lower in ACJ $(9 \%)$ and AWA $(5.3 \%)$ feeds compared with the remaining diets (16-24\%) (Fig. 1A). At genus or lowest taxonomic rank, the ACJ and AWA feeds were dominated by Pediococcus (62\%) and Bacillaceae (68\%), respectively (Fig. 1B). On the contrary, the microbiota composition in FM, ICJ, IWA and SBM feeds were dominated by Lactobacillus (21-25\%), Limosilactobacillus (22-25\%), Photobacterium (15-22\%), HTOO2 (10-11\%) and Ligilactobacillus (6.7-7.7\%) (Fig. 1B).

The microbiota in the source water was dominated by phyla Proteobacteria (55\%), Actinobacteriota (14\%) and SAR324 clade (Marine group B) (14\%), whereas the taxonomic compositions of the rearing tank water were dominated by phyla Proteobacteria (55\%) and Bacteroidota $(31 \%)$ (Fig. S2A). At the genus or lowest taxonomy level, SUP05 cluster (13\%), Candidatus Actinomarina (10\%) and Clade II $(9 \%)$ dominated the microbiota in the source water (Fig. S2B). The microbiota in the rearing tank water were dominated by the taxa Sulfitobacter (11\%), Colwellia (7\%), Hellea (7\%), Lacinutrix (5\%) and Maribacter $(5 \%)$ (Fig. S2B). Bacillaceae (0.01 - 0.2\%) and Pediococcus $(0.02$ $-2 \%$ ) were detected in both source water and tank water.

Digesta-associated microbiota. Regardless of the diets, the taxonomic compositions of the digesta samples at phy- lum level were dominated by Firmicutes, Proteobacteria and Actinobacteriota (Fig. 2A). Fish fed ACJ (97\%), and AWA (97\%) had higher abundance of Firmicutes compared with those fed the other diets (76-81\%) (Fig. 2A). Conversely, fish fed ACJ $(2.5 \%)$ and AWA (2.2\%) diets had lower composition of Proteobacteria compared with fish fed the other diets (12-19\%) (Fig. 2A). Actinobacteriota composition in the digesta of fish fed ACJ (0.2\%) and AWA (0.4\%) diets was lower compared with fish fed the remaining diets (3.3-4.1\%) (Fig. 2A).

The taxonomic composition of digesta samples at the genus or lowest taxonomy rank was influenced by the dietary group (Figs. 2B \& 3). Fish fed ACJ (92\%) diet were significantly dominated by Pediococcus compared with the other diets (Figs. 2B \& 3). Similarly, fish fed AWA (88\%) diet were significantly dominated by Bacillaceae compared with fish fed the other diets (Figs. 2B \& 3). Lactobacillus (12\%) and Limosilactobacillus (21\%) were significantly higher in fish fed FM compared with fish fed the other diets (Figs. 2B \& 3). Fish fed ICJ, IWA and SBM diets (5.4-6.3\%) had significantly higher abundant of Enterococcus compared with the other diets (Figs. 2B \& 3). Streptococcus, Peptostreptococcus, HT002, RsaHf231, Weissella and Photobacterium were significantly higher in fish fed FM, ICJ, IWA and SBM diets compared with fish fed ACJ and AWA diets (Figs. 2B \& 3).

When comparing the ASVs of the gut, water and feed, the composition of the gut microbiota was similar to that of the feed, but different from the water microbiota (Fig. 4). The ASVs overlap between the gut and the feed was higher than between the gut and water.

Core microbiota. In total, 94 ASVs were identified as core microbiota (present in $80 \%$ of the digesta samples) in fish fed the experimental diets (Fig. S3A-B; Table S2). Fifteen ASVs classified as Peptostreptococcus, Limosilactobacillus, Weissella, Ligilactobacillus, Streptococcus and Lachnospiraceae were identified to be present in all the dietary groups. Fish fed FM and SBM diets shared 37 primary core ASVs, belonging to members of Peptostreptococcus, Photobacterium, RsaHf231, and lactic acid bacteria (LAB) including Streptococcus, Lactobacillus, Limosilactobacillus, Weissella, Ligilactobacillus and HTOO2.

Alpha-diversity. Based on the four indices, the microbial diversity of fish fed ACJ and AWA diets was significantly lower compared with fish fed the other diets (Fig. 5; Table S3). The observed ASVs and Faith's PD showed that fish fed FM diet had significantly higher microbial diversity compared with fish fed ICJ, IWA and SBM diets (Figs. 5A, D). Contrarily, based on Shannon's index, the microbial diversity of fish fed FM diet was significantly lower compared with those fed ICJ, IWA and SBM diets (Fig. 5C). Excluding fish fed ACJ and AWA diets, the microbial diversity was similar among the other diets based on Pielou's evenness (Fig. 5B). The microbial compositions of fish fed ICJ, IWA and SBM were similar based on the four alpha-diversity indices (Fig. 5). 

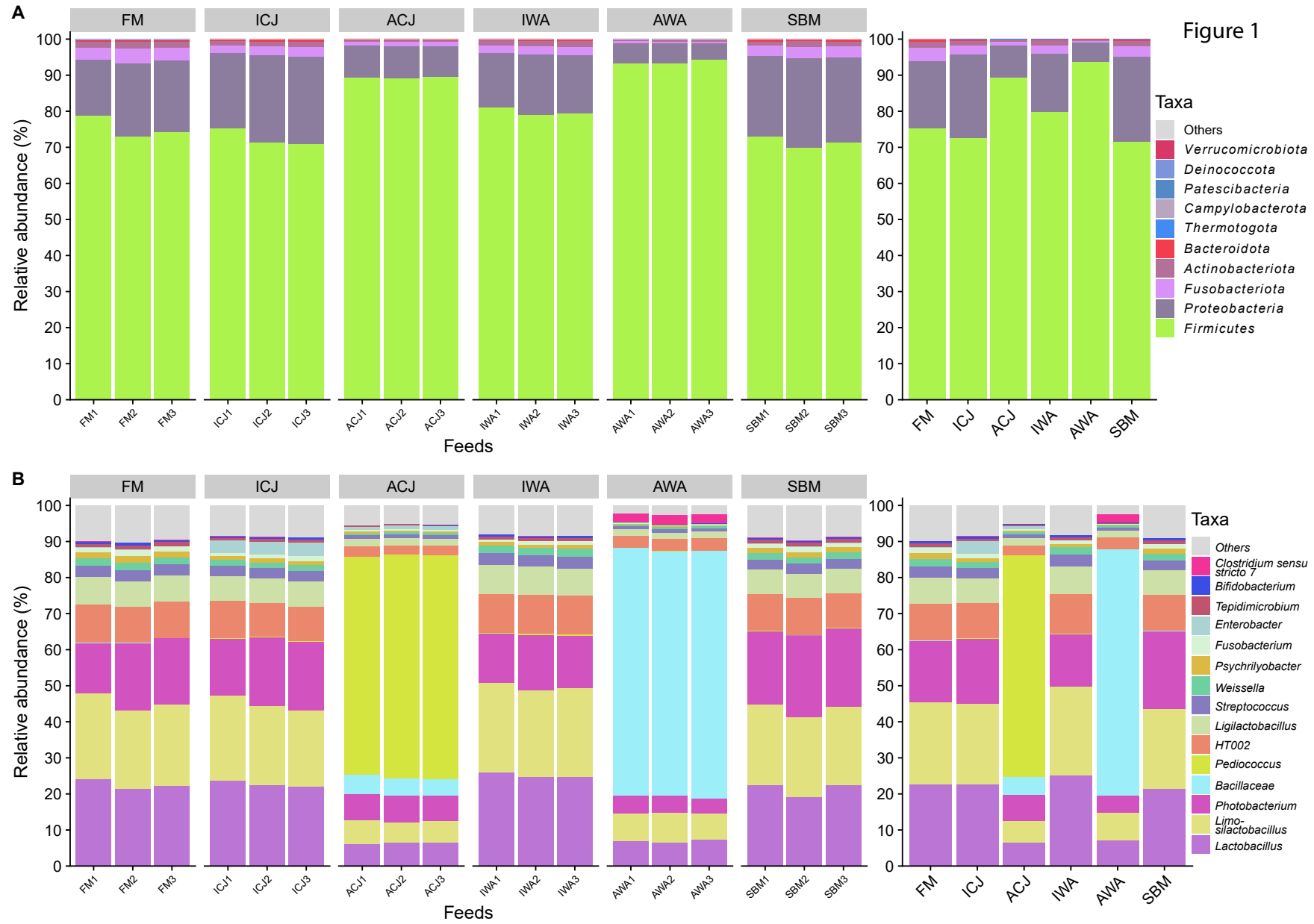

Fig. 1. Microbiota composition in the feed samples. Relative abundance of the top 10 most abundant taxa at phylum level (A) and top 15 most abundant taxa at genus or lowest taxonomic rank (B). The mean relative abundance of each taxon within the same diet is displayed on the right side. The samples are grouped by diets; FM fishmeal-based; SBM - soybean meal-based; 4 experimental diets containing $300 \mathrm{~g} / \mathrm{kg} \mathrm{SBM}$ and $100 \mathrm{~g} / \mathrm{kg}$ of ICJ - inactivated Cyberlindnera jadinii; ACJ - autolyzed C. jadinii; IWA - inactivated Wickerhamomyces anomalus; AWA - autolyzed W. anomalus diets.

Beta-diversity. The PCoA plots built on the four betadiversity indices showed that the microbiota of fish fed FM diet were clearly distinct from the other diets (Fig. 6). Based on the four beta-diversity indices, the PCoA plots showed that microbiota of fish fed ICJ, IWA and SBM diets were similar, and clearly clustered from those fed FM, ACJ and AWA diets (Fig. 6A-D). The PCoA plots based on Jaccard distance, unweighted UniFrac distance and PhILR transformed Euclidean distances showed separation of microbiota in fish fed ACJ diet compared with fish fed AWA diet (Fig. 6A, B, D). On the contrary, the microbiota of fish fed ACJ diet were similar compared with fish fed AWA diet based on Aitchison distance matrix (Fig. 6C). The PERMANOVA tests showed that beta-diversity were significantly influenced by the dietary groups, and the results were in line with the PCoA plots (Table S4). Based on the four distance matrices, the microbiota of fish fed FM diet were significantly different from those fed the other diets. Also, the PERMANOVA tests showed similarity in the microbiota of fish fed ICJ, IWA and SBM diets, which were different from those fed ACJ and AWA diets. The statistical tests showed that the microbiota of fish fed ACJ diet were significantly different from fish fed AWA diet. The tests for homogeneity and multivariate disper- sions are presented in Fig. S4 and Table S5. The multivariate dispersions were significantly affected by the dietary groups based on the four distance matrices.

Metabolic capacity of gut microbiota. Fifty-eight percent (526) of the 906 ASVs identified in the current study could be mapped to at least one model from a published collection of GSMMs of gut microbiota. Thirty-seven percent (338), $19 \%$ (176) and $1.3 \%$ (12) of the ASVs were matched to family, genus, and species, respectively (Fig. S5A). The ASVs matched to family, genus, and species were mapped to an average of 16, 13 and 1 model(s), respectively (Fig. S5B). The models mapped to ASVs contained 4802 different reactions, half of which $(55 \%)$ were present in all samples. Most samples $(90 \%)$ contained more than $90 \%$ of the reactions, but the abundances of many reactions differed significantly between samples and diets. Furthermore, the variability in the data could be explained in a few components using PCA of reaction abundances rather than ASV abundances (Figs. S6 and S7).

By classifying the reactions into metabolic pathways, ten pathways were enriched in pairwise comparisons between the dietary groups (Fig. 7). The differences in mean abun- 

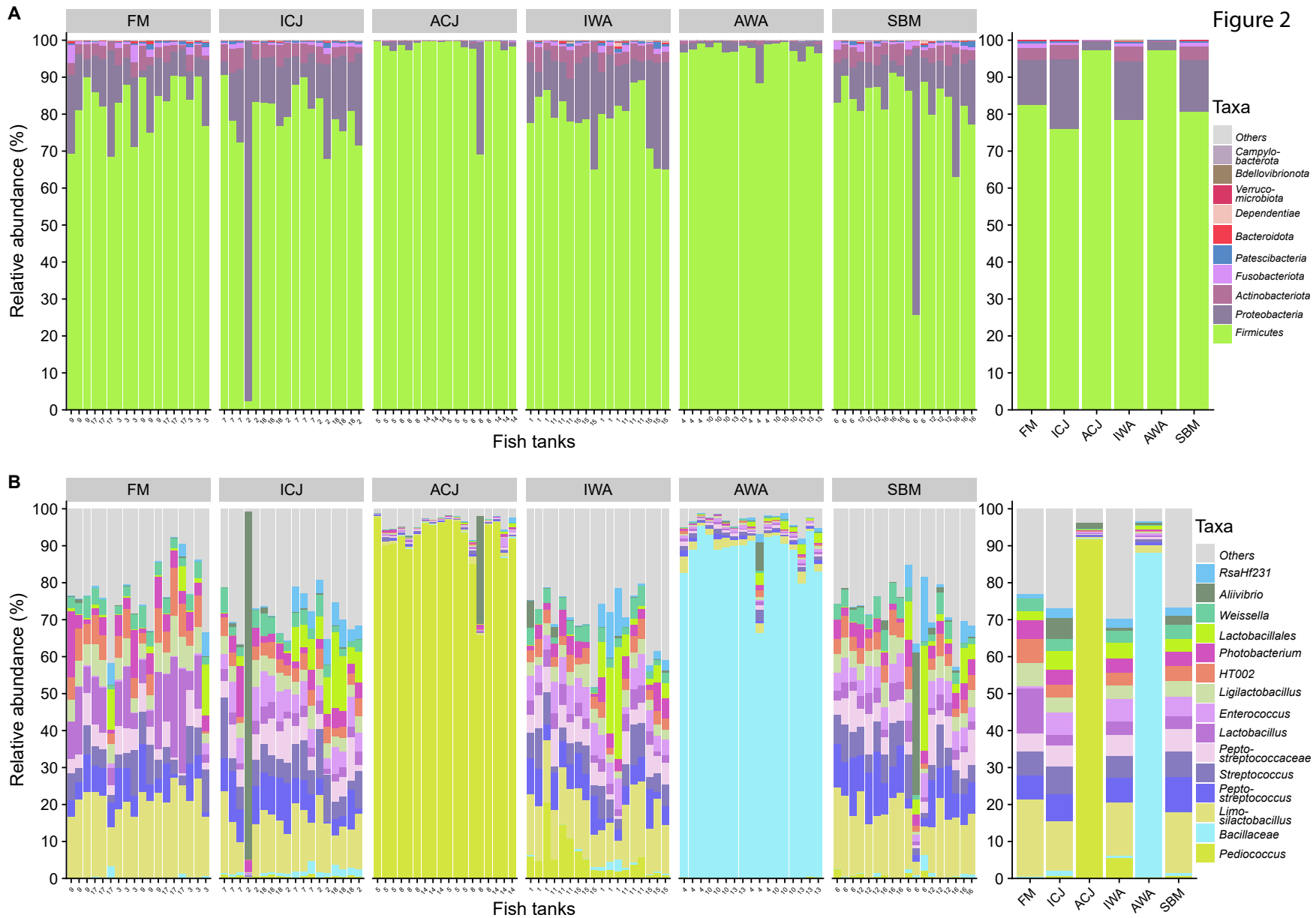

Fig. 2. Microbiota composition in the digesta of fish fed the experimental diets. Relative abundance of the top 10 most abundant taxa at phylum level (A) and top 15 most abundant taxa at genus or lowest taxonomic rank (B). The mean relative abundance of each taxon within the same diet is displayed on the right side. The samples are grouped by diets; FM - fishmeal-based; SBM - soybean meal-based; 4 experimental diets containing $300 \mathrm{~g} / \mathrm{kg}$ SBM and $100 \mathrm{~g} / \mathrm{kg}$ of ICJ - inactivated Cyberlindnera jadinii; ACJ - autolyzed C. jadinii; IWA - inactivated Wickerhamomyces anomalus; AWA - autolyzed W. anomalus diets.

dance of enriched pathways for each pair of diets are presented in Fig. S8. The gut microbiota of fish fed FM diet showed predicted enrichment of metabolic pathways related to mucin O-glycan degradation, valerate metabolism and OGlycan degradation, as well as lower enrichment of purine and pyrimidine catabolism pathways compared with fish fed ICJ and SBM diets (Figs. $7 \&$ S8A, E). The gut microbiota of fish fed ACJ diets showed predicted enrichment of mucin O-glycan degradation pathway compared with fish fed ICJ, IWA, AWA and SBM diets (Figs $7 \&$ S8). The predicted enrichment of metabolic pathways was similar for fish fed FM and ACJ diets, except for glycerophospholipid pathway (enriched in fish fed FM) and nucleotide interconversion (enriched in fish fed ACJ) (Figs $7 \&$ S8B).

\section{Discussion}

Core microbiota. In line with previous studies (27-29), Limosilactobacillus, Weissella, Ligilactobacillus and Streptococcus were annotated as core microbiota in the present study. Limosilactobacillus, Weissella, Ligilactobacillus and Streptococcus are commonly identified in the intestine of Atlantic salmon reared in seawater $(23,27,30)$. These taxa belong to the group of lactic acid bacteria (LAB), which are known to promote beneficial health effects in fish (3133). The environmental factors (e.g., feeds) before seawater transfer possibly influenced the colonization of these microbiota in the fish gut. Peptostreptococcus and Lachnospiraceae were also identified as core taxa in the present study. These taxa have been found in the intestinal digesta of Atlantic salmon but are rarely identified as core microbiota $(27,28,30)$. Lachnospiraceae are associated with production of short chain fatty acids (butyrate) (34), and has been reported to play a role in preventing inflammatory diseases in fish (35). It is noteworthy to state that, Mycoplasma which is commonly reported as core microbiota in the intestine of both wild and farmed Atlantic salmon $(29,30,36-41)$, was not identified in the present study. It is unclear why Mycoplasma was not detected, but it might be linked to the differences in environmental factors during the early life stages of fish such as, live food, feeds, water temperature and salinity or simply lack of exposure to Mycoplasma. These factors are reported to influence the establishment of core microbiota in fish (31, 39-43). Also, a recent study has demonstrated that the establishment of Mycoplasma increased with time in seawater (41), implying that the experimental duration may be 

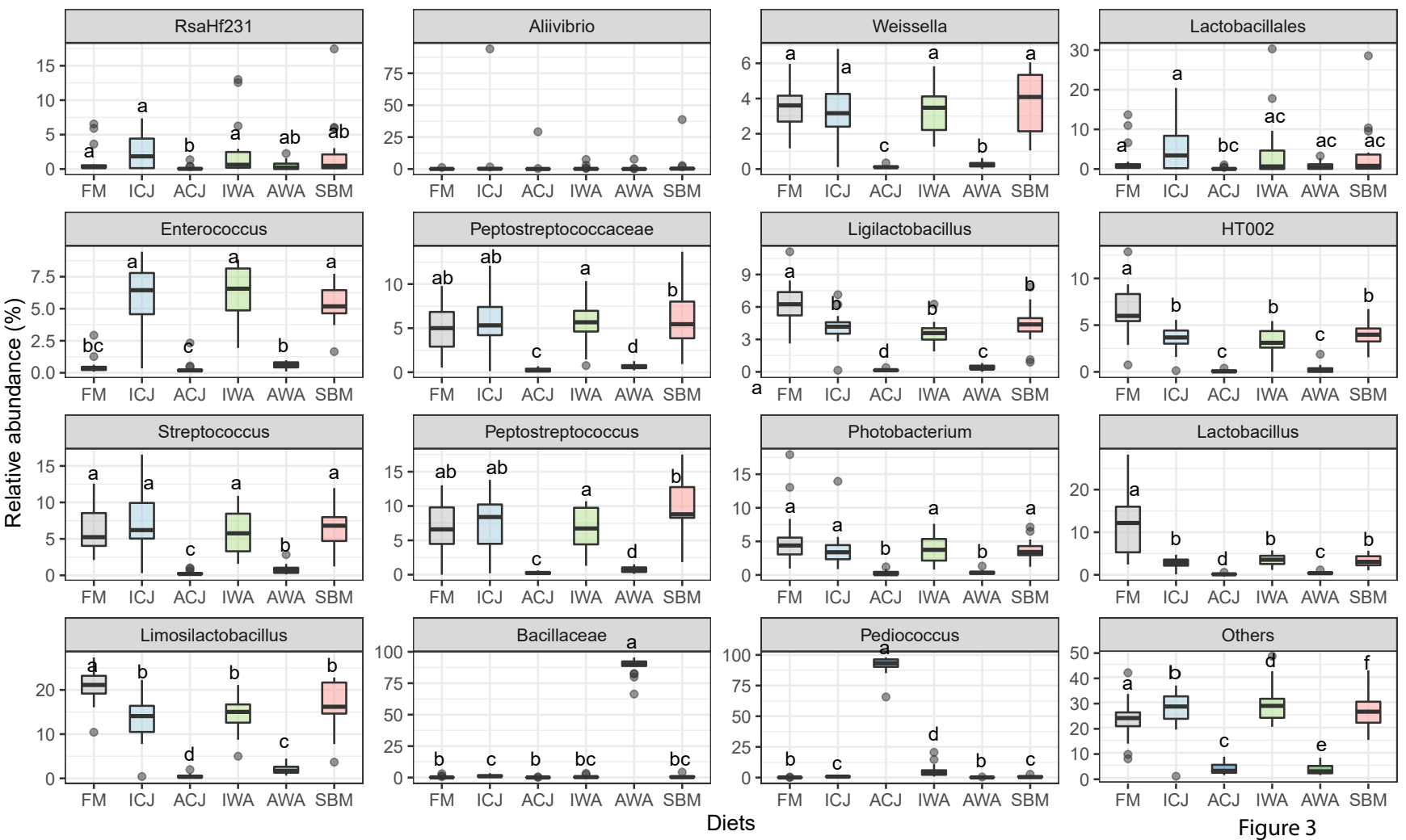

Fig. 3. Boxplots of relative abundance of the top 15 most abundant taxa (at genus or lowest taxonomic rank) in the digesta of fish fed the experimental diets. The samples are grouped by diets; FM - fishmeal-based; SBM - soybean meal-based; 4 experimental diets containing $300 \mathrm{~g} / \mathrm{kg}$ SBM and $100 \mathrm{~g} / \mathrm{kg}$ of ICJ - inactivated Cyberlindnera jadinii; ACJ - autolyzed C. jadinii; IWA - inactivated Wickerhamomyces anomalus; AWA - autolyzed W. anomalus diets. Different lower-case letters represent taxa with significantly different $(\mathrm{p}<0.05)$ relative abundance among the diets.

a

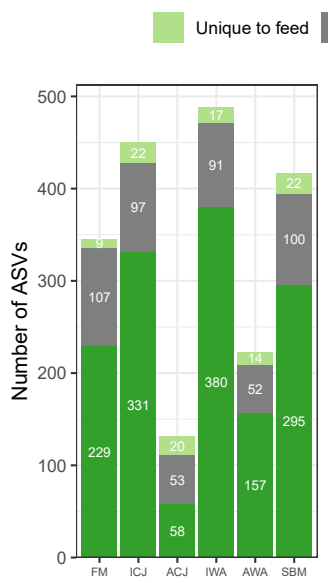

b

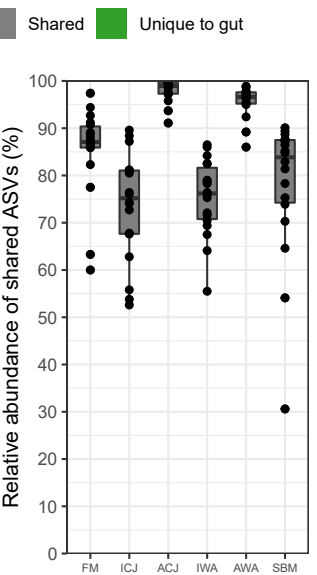

Figure 4

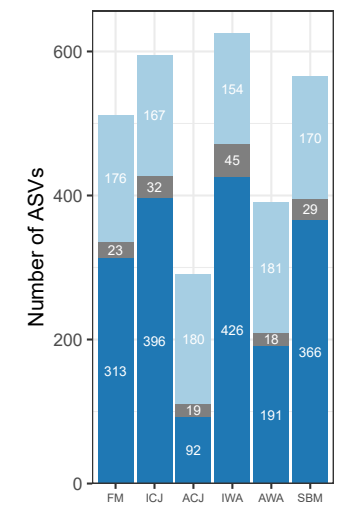

Shared Unique to gut

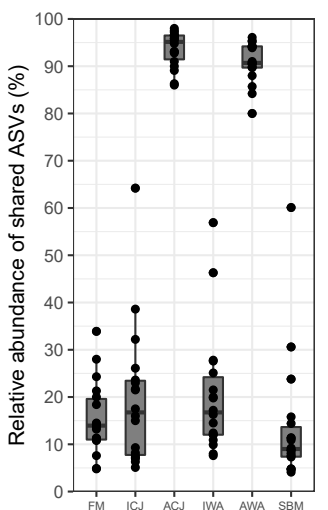

Fig. 4. The microbial overlap between the gut and feeds (a) and between the gut and the water (b). The number of shared amplicon sequence variants (ASVs) is shown in the left figure of each panel. The relative abundance of shared ASVs is shown in the right figure of each panel. The minimum relative abundance of ASVs to be considered as present in a sample was $0.05 \%$. The samples are grouped by diets; FM - fishmeal-based; SBM - soybean meal-based; 4 experimental diets containing $300 \mathrm{~g} / \mathrm{kg}$ SBM and $100 \mathrm{~g} / \mathrm{kg}$ of ICJ - inactivated Cyberlindnera jadinii; ACJ - autolyzed C. jadinii; IWA - inactivated Wickerhamomyces anomalus; AWA - autolyzed W. anomalus diets.

too short for its establishment in the gut of fish used in the current experiment.

Soybean meal has a dominating effect on modulation of gut microbiota. In accordance with previous findings in fish (23-26, 44-46), the present study observed differences between the gut microbiota of fish fed FM diet compared with those fed SBM diet. The microbial richness and diversity were higher in fish fed FM diet compared with fish fed SBM diet, which is in line with previous studies $(23,24)$. Most of the microbial taxa found in Atlantic salmon gut such as Lactobacillus, Limosilactobacillus, Ligilactobacillus, HTOO2, and Vagococcus were more abundant in fish fed 

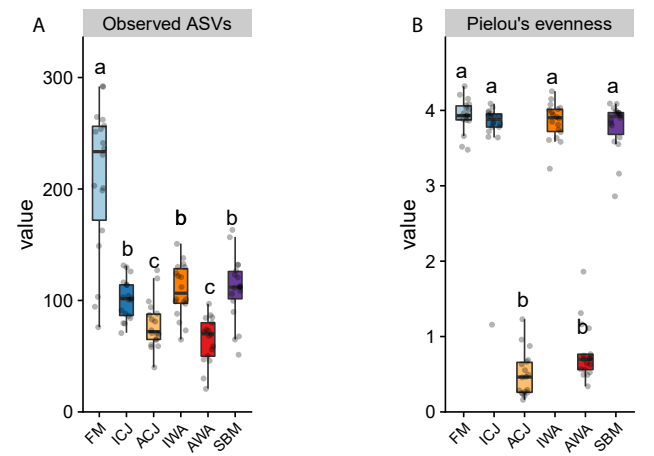

Figure 5
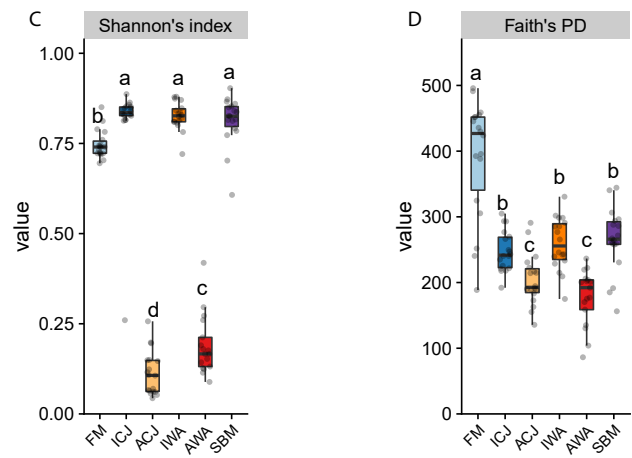

Fig. 5. Boxplots of alpha-diversity of gut microbiota of fish fed the experimental diets. The four alpha-diversity indices used are; (A) observed amplicon sequence variants (ASVs), (B) Pielou's evenness (C) Shannon's index and (D) Faith's phylogenetic diversity (PD). The samples are grouped by diets; FM - fishmeal-based; SBM soybean meal-based; 4 experimental diets containing $300 \mathrm{~g} / \mathrm{kg}$ SBM and $100 \mathrm{~g} / \mathrm{kg}$ of ICJ - inactivated Cyberlindnera jadinii; ACJ - autolyzed C. jadinii; IWA - inactivated Wickerhamomyces anomalus; AWA - autolyzed W. anomalus diets. Indices with different lower-case letters are significantly different $(\mathrm{p}<0.05)$ among the diets.
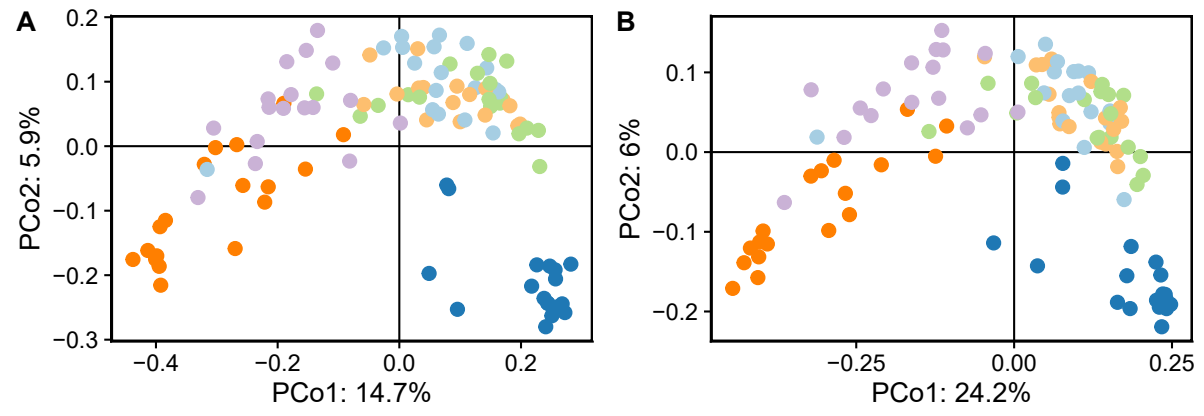

Figure 6
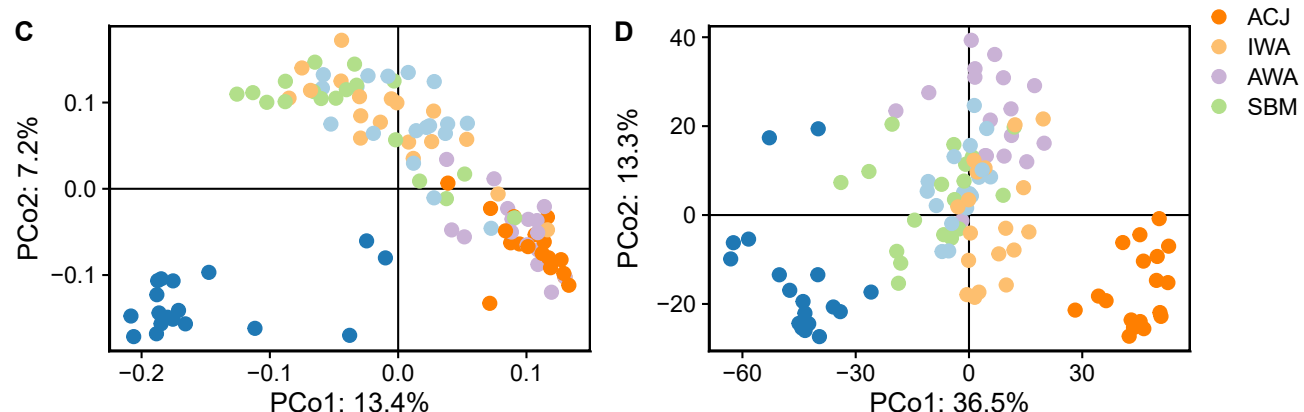

Fig. 6. Principal coordinates (PCo) analysis plots of beta-diversity of gut microbiota of fish fed the experimental diets. The four beta-diversity indices used are; (A) Jaccard distance, (B) Unweighted Unifrac distance (C) Aitchison distance and (D) PhILR transformed Euclidean distance. The samples are grouped by diets; FM fishmeal-based; SBM - soybean meal-based; 4 experimental diets containing $300 \mathrm{~g} / \mathrm{kg}$ SBM and $100 \mathrm{~g} / \mathrm{kg}$ of ICJ - inactivated Cyberlindnera jadinii; ACJ - autolyzed C. jadinii; IWA - inactivated Wickerhamomyces anomalus; AWA - autolyzed W. anomalus diets.

FM diet compared with fish fed SBM diet. The current results showed that the microbiota of fish fed SBM were dominated by LAB such as Lactobacillus, Limosilactobacillus, Ligilactobacillus, Weissella, Enterococcus and Streptococcus, which is in accordance with previous findings (23-25). The high abundance of LAB in fish fed SBM-based diet has been attributed to the presence of soluble and insoluble oligosaccharides such as raffinose and stachyose, which can be used as substrates for metabolism and growth by the microbiota (23). Results from the present study published elsewhere (47) 


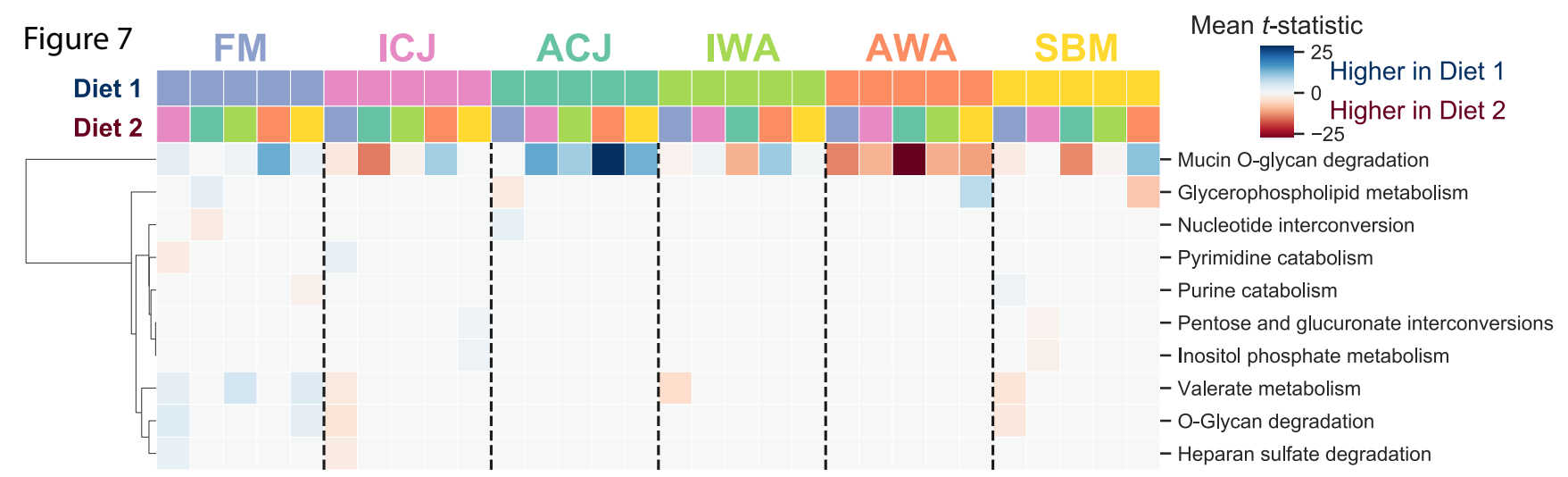

Fig. 7. Hierarchical clustering of the significantly enriched metabolic subsystems between each pair of dietary groups. Columns are diet pairs, rows are metabolic subsystem, and the color of each cell indicates whether the metabolic subsystem was enriched in diet 1 (blue) or diet 2 (red). The samples are grouped by diets; FM fishmeal-based; SBM - soybean meal-based; 4 experimental diets containing $300 \mathrm{~g} / \mathrm{kg} \mathrm{SBM}$ and $100 \mathrm{~g} / \mathrm{kg}$ of ICJ - inactivated Cyberlindnera jadinii; ACJ - autolyzed C. jadinii; IWA - inactivated Wickerhamomyces anomalus; AWA - autolyzed W. anomalus diets.

showed that fish fed SBM diet developed typical signs of SBMIE. As previously mentioned, LAB are generally considered as beneficial microbes promoting intestinal health and growth of fish. Although members of LAB, such as some species of Enterococcus and Streptococcus, are considered pathogenic, it seems counterintuitive that LAB enrichment could be observed in fish that developed SBMIE. This observation challenges the general understanding that microbiota play a role in the development of SBMIE in fish. The relationship between increased relative abundance of LAB and development of SBMIE has been documented in previous studies $(23,24,26)$. Reveco et al. (24) speculated that the increased relative abundance of $\mathrm{LAB}$ could be related to their capability to produce antimicrobial peptides (such as bacteriocins) against the certain bacteria in fish presenting SBMIE. Also, during the development of SBMIE, it is possible that the commensal bacteria (LAB) have less competition and more opportunity to proliferate. It remains unclear whether the increase in relative abundance of LAB is a cause or a consequence of the inflammatory response in fish presenting SBMIE. Further investigation is needed to clarify the role of intestinal microbiota in the development of SBMIE in Atlantic salmon fed plant-based diets.

The present study revealed that microbial richness and diversity were similar among fish fed ICJ, IWA and SBM diets. This implies that the inclusion of inactivated yeasts (CJ and WA) did not modulate the intestinal microbiota of fish fed SBM diet. This contradicts previous findings which showed that feeding diets containing Saccharomyces cerevisiae and WA yeasts modulated the intestinal microbiota in rainbow trout $(48,49)$. It is worthy of note that SBM was not used in the previous studies $(48,49)$. In line with our present results, dietary supplementation of mannan oligosaccharides (MOS) from yeasts did not modulate microbial diversity and richness of gilthead sea bream fed SBM-based diet (50). These contradicting results underscore the importance of ingredients used in diet formulation with respect to possible effects of yeast or its cell wall components on gut microbiota of fish (51). The cell wall polysaccharides of yeasts such as glucans and MOS can serve as substrates for microbial growth (52-54), and as a consequence modulates the intestinal microbiota in fish fed yeast-based diets $(48,49)$. However, our speculation is that $30 \%$ inclusion level of SBM possibly has a dominating effect in modulating gut microbiota when compared with $10 \%$ inclusion level of inactivated yeasts in the current study. Study on the effects of inactivated yeasts (CJ and WA) in Atlantic salmon fed SBM-free diets is recommended in the future. Despite the similarity in microbial composition of fish fed ICJ, IWA and SBM diets, the results of the present study reported elsewhere (47) showed that inclusion of inactivated yeasts ( $\mathrm{CJ}$ and $\mathrm{WA}$ ) dampened the inflammatory response in the distal intestine of fish fed SBM diet. Therefore, it can be hypothesized that the ameliorating effects of inactivated yeasts on SBMIE is related to their capability to stimulate immune responses rather than through modulation of intestinal microbiota in Atlantic salmon.

Autolyzed yeasts modulate gut microbiota of fish. The results of the present study revealed that the gut bacteria composition of fish fed ACJ and AWA diets were greatly affected by the diets when compared with the other groups. The ACJ and AWA diets promoted the dominance of genus Pediococcus and the family Bacillaceae, respectively. Such modulation consequently led to a decrease in richness and diversity of gut microbiota of fish fed ACJ and AWA diets compared with fish fed the remaining diets. A previous study reported that autolyzed $S$. cerevisiae reduced the microbial diversity of gilthead sea bream fed commercial-like diet (55).

The increased relative abundance of Pediococcus and Bacillaceae in Atlantic salmon fed the autolyzed yeasts may be explained by the autolytic conditions, feed-borne microbiota and/or feed composition. Based on BLAST analysis using the NCBI database, the Pediococcus ASV in our data set revealed sequence homologous to Pediococcus acidilactici and P. claussenii, whereas the Bacillaceae ASVs matched a wide range of members in the Bacilli microbial clade, including Caldibacillus pasinlerensis, C. thermoamylovorans, Cerasibacillus terrae, C. quisquiliarum, Alkalihalobacillus gibsonii and A. lonarensis. Optimum growth temperature for the genus Pediococcus (56) and the family Bacillaceae 
(57) ranged between $30-60{ }^{\circ} \mathrm{C}$. Thus, it is plausible that the growth of spores of these microbial taxa were selectively promoted during the autolytic process (at $50{ }^{\circ} \mathrm{C}$ for $16 \mathrm{~h}$ ). Although thermal condition during the spray-drying was expected to inactivate the microbes in the yeast, dead or bacterial spores can still be profiled by the DNA sequencing methods. We could assert that the inclusion of autolyzed yeasts promotes the enrichment of a certain microbial taxon in the digesta of fish, but the effects seem to be yeast dependent. Therefore, the observed dominance of these microbial taxa in the gut of fish fed ACJ and AWA feeds probably reflects not only active microbes, but also dead microbes and spores transferred from the yeasts into the feeds. In future studies, analyzing the microbes in the yeast cream and the dried yeasts would further elucidate the extent to which the diet effects are attributable to the transfer of microbes from the yeasts to the diets. Techniques such as viability PCR and RNA sequencing (58), which are able to distinguish dead or active microbes, would provide useful information regarding the role of yeast- and feed-associated microbes in shaping the intestinal microbiota of fish fed yeast-based diets. Changes in cell wall polysaccharide of autolyzed yeasts may also partly contributed to the observed dominance of Pediococcus and Bacillaceae in fish fed ACJ and AWA diets. Previous studies have reported that the solubility (59) and biophysical properties $(18,60)$ of cell wall polysaccharides of yeasts are modified by the autolytic process. It is possible that the glucans and MOS in autolyzed yeasts are more available as substrates for the intestinal microbiota compared with intact yeasts. In the current study, it was impossible to distinguish whether the substrates for microbiota growth and metabolism were derived from SBM or from the yeast. Thus, the extent to which the modification of cell wall polysaccharides of yeasts contributed to the intestinal microbiota of fish could not be ascertained. This hypothesis can be tested by supplementing autolyzed yeasts to SBM-free diets and sequencing the intestinal microbiota of fish fed these diets.

It remains unclear whether the high abundance of a single taxon in fish fed ACJ or AWA diet was beneficial or caused dysbiosis in the host. The species P. acidilactici and Bacillus subtilis are among the most widely studied probiotic bacteria and have been reported to promote growth performance, nutrient digestion, disease resistance and intestinal health in farmed fish (20,61-64). Based on this, it was expected that the high relative abundance of Pediococcus and Bacillaceae in fish fed the autolyzed yeasts would enhance the performance and intestinal health compared with fish fed the other diets. This was not the case, based on the results of fish performance and intestinal health presented in Agboola et al. (47). Fish performance was unaffected by the dietary treatments, and the inclusion of autolyzed yeasts in fish fed SBM did not alleviate SBMIE beyond the level observed for fish fed SBM with inactivated yeasts (47). Therefore, it is possible that the physiological response of fish to high relative abundance of both Pediococcus and Bacillaceae is limited by low feed intake and short experimental period used in the current study. Long-term experiments with ad-libitum fish feeding of diets containing autolyzed yeasts is recommended in the future. Also, it could simply be that the microbiota are dead and without probiotic effects in fish. The lack of difference in physiology of fish fed inactivated and autolyzed yeasts also supports the hypothesis that the dominance of a single taxon in the gut of fish fed ACJ and AWA is due to transfer of bacteria spores from the feeds to the fish gut. Thus, the reproducibility of microbiota modulated in fish fed yeast-based diets in the present study should be investigated in future studies. It is important to note that no mortality or noticeable signs of disease were recorded, suggesting that the high abundance of a single taxon in fish fed ACJ and AWA diets in the present study did not lead to dysbiosis.

Gut microbiota is driven by feed microbiota and less by water microbiota. Feed and rearing water are two environmental factors shaping the intestinal microbiota of fish (65-74). In agreement with previous studies in fish (65-70), there was high overlap between microbiota in the gut and the feeds. Still, it is unclear to what extent the carry-over microbes from the feeds influenced the intestinal microbiota. It would be interesting in the future to stain for live/dead gut bacteria and then use fluorescence-activated cell sorting followed by $16 \mathrm{~S}$ rRNA sequencing to identify the dead spores from the live bacteria. In the current study, microbial overlap between the intestine and the feeds was higher than the microbial overlap reported elsewhere $(70,75)$ in Atlantic salmon fed insect-based diets. The discrepancy can be attributed to the feed processing technology used in these studies. Contrary to the present study, feeds used in the previous studies $(70,75)$ were processed using extrusion technology. Extrusion is a hydrothermal process that is capable of inactivating microbes, thus, it is likely that the viability of feed microbes in this study was higher than the previous studies $(70,75)$. This may be responsible for the higher microbial overlap between the feeds and the intestine in the current study compared with earlier studies. However, it is reported that the feed processing (pre-conditioning vs. nonpreconditioning) slightly influenced the gut microbiome of rainbow trout (76). Further investigation on the impact of extrusion treatment on intestinal microbiota of fish fed yeastbased diets in Atlantic salmon may be needed in the future. In accordance with previous studies $(25,75)$, water had a lower impact in shaping the intestinal microbiota of fish than the feeds. Microbial overlap between water and the intestine in the current study was higher than reported for Atlantic salmon reared in freshwater $(25,70,75)$. In seawater, Atlantic salmon maintain osmoregulation by ingesting water to compensate for water loss to the hyperosmotic environment (77). Water drinking ability of salmon reared in seawater may facilitate uptake of microbes, and thus, be responsible for the higher microbial overlap between water and the intestine compared with previous studies in freshwater phase $(25,70,75)$.

Metabolic capacity of gut microbiota. The gut microbiota plays a critical role in host physiology by supporting growth performance, nutrient digestion, metabolism and par- 
ticipating in immune system maturation and pathogen defense $(78,79)$. In the current study, a metagenome prediction tool was used to investigate the metabolic capacity of the gut microbiota of fish fed the experimental diets. The results revealed that the gut microbiota of fish fed ACJ diet were enriched in pathways related to mucin O-glycan degradation compared with fish fed the other diets. The gut microbiota of fish fed ACJ was dominated by Pediococcus, which has capability to adhere to intestinal mucus (80) and intestinal epithelial cells (81). The breakdown of mucin glycans by the gut microbiota generates a pool of microbial products that can be beneficial for host mucus production and for immune and metabolic responses $(21,22)$. This plays an important function in mucosal health, which is considered the first line of defense protecting the epithelial layer from pathogen invasion and other luminal compounds (21). Our results further showed that pathways related to valerate metabolism were enriched in fish fed FM diet compared with fish fed ICJ, IWA and SBM diets. Valerate is a scarcely studied short chain fatty acid that can be produced as an end product of microbial fermentation (82). The production of short chain fatty acids can act as link between the microbiota and the immune system by modulating the different aspects of intestinal epithelial cell $(82,83)$. It has been reported that valerate production can help to inhibit the growth of Clostridioides difficile, both in vitro and in vivo (84), a bacterium that has been implicated in the development of inflammatory bowel disease in humans (85). Although the role of valerate on fish physiology is not reported in literature, it is possible that increased valerate metabolism may be responsible for the normal intestinal health observed in fish fed FM diet in the current study (47).

Prediction tools are used to infer metabolic functions of gut microbiota produced through amplicon sequencing (8688 ), but their validity is often questionable (88). The GSMMs used in the current study were based on human gut microbiota, and the predicted metabolic capacities may not exactly mimic that of fish gut microbiota. Additionally, only about half of the identified ASVs were matched to a known GSMM, thus limiting the ability of the analysis to represent the whole gut microbiota of fish used in the present study. Based on these shortcomings, the results of the predicted metabolic capacity of fish gut microbiota reported in this study should be interpreted with caution.

\section{Conclusions}

The present study showed that the richness and diversity of gut microbiota was lower in fish fed SBM compared with fish fed FM diet. The microbial composition and richness were similar among fish fed ICJ, IWA and SBM diets. Inclusion of autolyzed yeasts (ACJ and AWA) lowered the richness and diversity of gut microbiota in fish. Fish fed ACJ diet increased relative abundance of Pediococcus, and mucin O-glycan degradation pathway while fish fed AWA diet increased relative abundance of Bacillaceae compared with other diets. The results also suggest that the ameliorating effects of yeasts on SBMIE is related to their capability to stimulate immune cells rather than through modulation of intesti- nal microbiota in Atlantic salmon. Future research should focus on increasing our understanding of functional role of microbiota enhanced through inclusion of yeasts in fish diets

\section{Methods}

Yeasts, experimental diets, and fish feeding trial. The CJ and WA yeast biomass were produced in a $30 \mathrm{~L}$ bioreactor using a growth medium composed of a blend of enzymatic hydrolysates of pre-treated spruce wood (Picea abies) and chicken by-products as described by Lapeña et al. (13). After harvesting, the yeasts were processed following the protocol described by Agboola et al. (18). Briefly, the yeast biomass was washed, centrifuged and the resulting paste was divided into two equal parts. One part of the yeast paste was directly inactivated with a spray-dryer (SPX 150 MS, SPX Flow Technology, Denmark) set at $180{ }^{\circ} \mathrm{C}$ and $80{ }^{\circ} \mathrm{C}$ for inlet and outlet temperature, respectively. The other half of the yeast paste was autolyzed at $50{ }^{\circ} \mathrm{C}$ for $16 \mathrm{~h}$ in a stirred $30 \mathrm{~L}$ reactor (Einar, Belach Bioteknik, Sweden), followed by spray-drying using the same conditions as above. The resulting processed yeast products were: inactivated CJ (ICJ), autolyzed CJ (ACJ), inactivated WA (IWA), and autolyzed WA (AWA). The nutritional and cell wall compositions of the four yeast products are presented in Table S6.

Six experimental diets were formulated to meet or exceed $(89,90)$ the nutritional requirements of Atlantic salmon smolts; a fishmeal-based (FM) control diet, a challenging diet containing $30 \%$ soybean meal (SBM) and four diets containing $30 \%$ SBM with $10 \%$ inclusion of the different processed yeasts (ICJ, ACJ, IWA and AWA), respectively. Table 1 shows the ingredient and analyzed compositions of the six experimental diets. The diets were cold-pelleted using a P35A pasta extruder (Italgi, Carasco, Italy) and dried at 60 ${ }^{\circ} \mathrm{C}$ in small experimental driers. The production of the experimental diets is fully described in Agboola et al. (47).

A 42-day seawater feeding trial with Atlantic salmon smolts (initial body weight $=136 \pm 0.25 \mathrm{~g}$ ) was conducted at the research facility of the Norwegian Institute of Water Resources (NIVA, Solbergstrand, Norway). A total of 450 vaccinated salmon smolts were randomly allocated into 18 fiber tanks $(300 \mathrm{~L})$ and fed one of the six experimental diets ( $\mathrm{n}=3$ tanks per diet) for $6 \mathrm{~h}$ per day using automatic feeders delivering feed every 12 minutes. The fish were reared under a $24 \mathrm{~h}$ light regime in a flow-through system with an average water temperature of $11.5^{\circ} \mathrm{C}$ and average oxygen saturation of $84 \%$. The water flow was kept at an average of $5.5 \mathrm{~L} \mathrm{~min}^{-1}$ during the experimental period. Water salinity was gradually increased from $5 \mathrm{ppt}$ at the start, until it reached full salinity (33 ppt) during the first 12 days of the experiment.

Sample collection. At the end of the feeding trial, the average body weight of the fish was $179 \pm 7.06 \mathrm{~g}$. Six fish were randomly selected from each tank, anaesthetized with metacaine (MS-222, $50 \mathrm{mg} \mathrm{L}^{-1}$ water), and killed with a sharp blow to the head for digesta sampling. After dissection, the distal intestine was opened longitudinally and the digesta was carefully removed using sterile plastic spatulas. 
The digesta was placed in cryotubes, snap-frozen in liquid nitrogen and stored at $-80{ }^{\circ} \mathrm{C}$. To obtain sterile conditions, tools were cleaned and decontaminated using $70 \%$ ethanol and flaming between each fish. Additionally, feed and water samples were collected into sterile plastic containers and stored at $-80^{\circ} \mathrm{C}$. Water samples were collected from both the source tank and the fish rearing tanks.

DNA extraction. Total DNA was extracted from $200 \mathrm{mg}$ of digesta (18 samples per dietary group) and $100 \mathrm{mg}$ of ground feed (3 replicates per diet) using QIAamp ${ }^{\circledR}$ Fast DNA Stool Mini Kit (Qiagen, Hilden, Germany, Cat. No. 51604) following the manufacturer's specifications with some modifications as described elsewhere (75). In addition to the digesta and feed samples, total DNA was extracted from the water samples. $500 \mathrm{~mL}$ each of source water (2 samples) and rearing tank water (4 samples) were filtered through a MFMillipore membrane filter with $0.22 \mu \mathrm{m}$ pore size (SigmaAldrich, Cat. No GSWP04700) and total DNA was extracted using the same protocol described above. The rearing water $(500 \mathrm{~mL}$ from each tank) samples were mixed, and four sub-samples $(500 \mathrm{~mL}$ each) were taken and used for the DNA extraction. Total DNA was also extracted from blank filter paper used for the filtration of water samples. For quality control of the present workflow, a microbial community standard (mock), which consists of eight bacteria and two yeasts (ZymoBIOMICS ${ }^{\mathrm{TM}}$, Zymo Research, California, USA; Cat. No. D6300) was included for DNA extraction as positive control. In addition, a blank negative control was added to each batch of DNA extraction by omitting the input material. Total DNA were extracted from blank control, mock positive control and blank filter paper following the method used for digesta, feed and water samples. The DNA concentration of all the samples were measured in duplicates using Invitrogen ${ }^{\mathrm{TM}}$ Quant-iT $^{\mathrm{TM}}$ Qubit $^{\mathrm{TM}}$ dsDNA HS (High Sensitivity) assay kit (Thermo Fisher Scientific, California, USA, Cat. No. Q32854) with the Qubit 4 Fluorometer $\left(\right.$ Invitrogen ${ }^{\mathrm{TM}}$ ). The extracted DNA were stored at $-20^{\circ} \mathrm{C}$ until further analysis.

PCR amplification. The V3-V4 hypervariable regions of the bacterial 16S rRNA gene were amplified in a $25 \mu \mathrm{L}$ reaction volume containing 2x KAPA HiFi HotStart Ready Mix (12.5 $\mu \mathrm{L})$ (Roche Sequencing Solutions, Mat. No. 7958935001), DNA template $(5 \mu \mathrm{L})$, and $1.33 \mu \mathrm{M}$ primers $(3.75 \mu \mathrm{L}$ of each primer). The primers used for the amplicon PCR are $341 \mathrm{~F}$ (5'-CCT ACG GGN GGC WGC AG-3') and 785R (5'-GAC TAC HVG GGT ATC TAA TCC-3'). The amplification was set at initial denaturation of $95{ }^{\circ} \mathrm{C}$ for $3 \mathrm{~min} ; 25$ cycles of denaturation at $95{ }^{\circ} \mathrm{C}$ for $30 \mathrm{~s}$; annealing at $55{ }^{\circ} \mathrm{C}$ for $30 \mathrm{~s}$; extension at $72{ }^{\circ} \mathrm{C}$ for $30 \mathrm{~s}$; followed by a final extension at $72{ }^{\circ} \mathrm{C}$ for $5 \mathrm{~min}$. After the amplification process, duplicate PCR products were pooled and purified using Agentcourt AMPure XP beads (Beckman Coulter, Indiana, USA, Cat. No. A63881), and the cleaned PCR products were examined by $1 \%$ agarose gel electrophoresis.
Table 1. Diet formulation and nutritional composition of the experimental diets*.

\begin{tabular}{|c|c|c|c|c|c|c|}
\hline & FM & ICJ & ACJ & IWA & AWA & SBM \\
\hline \multicolumn{7}{|l|}{ Diet formulation ${ }^{\mathrm{a}}(\mathrm{g} / \mathrm{kg})$} \\
\hline Fishmeal $^{\text {b }}$ & 433.4 & 208.4 & 208.4 & 208.4 & 208.4 & 261.4 \\
\hline Soybean meal ${ }^{c}$ & 0 & 300 & 300 & 300 & 300 & 300 \\
\hline Wheat gluten meal $^{\mathrm{d}}$ & 170 & 111 & 111 & 111 & 111 & 136 \\
\hline Potato starch ${ }^{\mathrm{e}}$ & 120 & 68 & 68 & 68 & 68 & 90 \\
\hline Cellulose & 80 & 0 & 0 & 0 & 0 & 0 \\
\hline Yeast $^{\mathrm{n}}$ & 0 & 100 & 100 & 100 & 100 & 0 \\
\hline Fish oil ${ }^{\mathrm{f}}$ & 130 & 130 & 130 & 130 & 130 & 130 \\
\hline Gelating $^{\mathrm{g}}$ & 60 & 60 & 60 & 60 & 60 & 60 \\
\hline Monocalcium phosphate ${ }^{\mathrm{h}}$ & 0 & 10 & 10 & 10 & 10 & 10 \\
\hline Premix ${ }^{\mathrm{i}}$ & 5 & 5 & 5 & 5 & 5 & 5 \\
\hline L-lysine $\mathrm{e}^{\mathrm{j}}$ & 0 & 3 & 3 & 3 & 3 & 3 \\
\hline DL-Methionine $\mathrm{k}^{\mathrm{k}}$ & 0 & 3 & 3 & 3 & 3 & 3 \\
\hline Chlorine chloride ${ }^{1}$ & 1.5 & 1.5 & 1.5 & 1.5 & 1.5 & 1.5 \\
\hline Yttrium $^{\mathrm{m}}$ & 0.1 & 0.1 & 0.1 & 0.1 & 0.1 & 0.1 \\
\hline \multicolumn{7}{|c|}{ Diet composition (analyzed values) ${ }^{\circ}(\mathrm{g} / \mathrm{kg})$} \\
\hline Dry matter & 926.6 & 889.9 & 889.2 & 924.5 & 913.9 & 897.3 \\
\hline Crude protein & 531.8 & 518.3 & 530.3 & 519.5 & 521.4 & 542.6 \\
\hline Starch & 131.9 & 92.6 & 93.3 & 89.3 & 87.6 & 103.6 \\
\hline Ash & 78.3 & 74.7 & 74.8 & 73.7 & 73.5 & 77.2 \\
\hline Carbon & 509.1 & 502.5 & 517.8 & 513.1 & 511.0 & 509.7 \\
\hline Sulphur & 6.0 & 6.2 & 6.0 & 6.1 & 6.0 & 6.3 \\
\hline Energy (MJ/kg DM) & 23.3 & 23.3 & 23.3 & 23.1 & 23.1 & 23.1 \\
\hline $\mathrm{DP}: \mathrm{DE}^{\mathrm{p}}$ & 23.1 & 22.8 & 22.8 & 22.5 & 22.5 & 23.3 \\
\hline
\end{tabular}

${ }^{\mathrm{a}}$ Liet formulation are expressed in $\mathrm{g} / \mathrm{kg}$.
$\mathrm{b}$. AB, Panevezys, Lithuania; ${ }^{\mathrm{e}}$ Lygel F 60 , Lyckeby Culinar, Fjälkinge, Sweden; ${ }^{\mathrm{f}}$ NorSalmOil, Norsildmel, Egersund, Norway; ${ }^{\mathrm{g}}$ Rousselot 250 PS, Rousselot SAS, Courbevoie, France; ${ }^{\mathrm{h}}$ Monocalcium phosphate, Bolifor MCP-F, Oslo, Norway Yara; ${ }^{i}$ Premix fish, Norsk Mineralnæring AS, Hønefoss, Norway. Per kg feed ; Retinol 3150.0 IU, Cholecalciferol way Yara, ${ }^{2}$ Premix fish, Norsk Mineralnæring AS, Hønefoss, Norway. Per kg feed; Retino 3150.0 IU, Cholecalciferol
$1890.0 \mathrm{IU}, \alpha$-tocopherol SD $250 \mathrm{mg}$, Menadione $12.6 \mathrm{mg}$, Thiamin $18.9 \mathrm{mg}$, Riboflavin $31.5 \mathrm{mg}$, d-Ca-Pantothenate

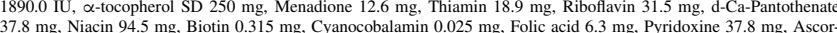
$37.8 \mathrm{mg}$, Niacin $94.5 \mathrm{mg}$, Biotin $0.315 \mathrm{mg}$, Cyanocobalamin $0.025 \mathrm{mg}$, Folic acid $6.3 \mathrm{mg}$, Pyridoxine $37.8 \mathrm{mg}$, Ascor-
bate monophosphate $157.5 \mathrm{~g}$. Cu: CuSulfate $5 \mathrm{H} 2 \mathrm{O} 6.3 \mathrm{mg}, \mathrm{Zn}:$ ZnSulfate $151.2 \mathrm{mg}$, Mn: Mn(II)Sulfate $18.9 \mathrm{mg}$. I: bate monophosphate $157.5 \mathrm{~g}$, Cu: CuSulfate $5 \mathrm{H} 2 \mathrm{O} 6.3 \mathrm{mg}$, Zn: ZnSulfate $151.2 \mathrm{mg}$, Mn: Mn(II)Sulfate $18.9 \mathrm{mg}$, I K-lodide $3.78 \mathrm{mg}$, Ca $1.4 \mathrm{~g}$; ${ }_{\mathrm{L}}$-Lysine CJ Biotech CO., Shenyang, China; ${ }^{\mathrm{K}}$ Rlot France; ${ }^{\mathrm{l}}$ Choline chloride, $70 \%$ Vegetable, Indukern SA., Spain; ${ }^{\mathrm{m}} \mathrm{Y}_{2} \mathrm{O}_{3}$. Metal Rare Earth Limited, Shenzhen, China.
${ }_{\mathrm{n}}^{\mathrm{n}}$ ICJ - inactivated Cyberlindnera jadinii; $\mathrm{ACJ}$ - autolyzed C. jadinii; IWA - inactivated Wickerhamomyces anomalus; ${ }^{\mathrm{n}} \mathrm{ICJ}$ - inactivated Cyberlindnera
$\mathrm{AWA}$ - autolyzed W. anomalus.

${ }^{\mathrm{O}}$ Diet composition are expressed in $\mathrm{g} / \mathrm{kg}$ dry matter (DM) unless otherwise stated.

$\mathrm{p}_{\mathrm{DP}} \mathrm{DE}=$ Digestible protein to digestible energy ratio. Calculated using internal digestibility values of various ingredi-

ents.
The diets are: FM - fishmeal-based; SBM - soybean meal-based; 4 other diets containing $300 \mathrm{~g} / \mathrm{kg}$ SBM and $100 \mathrm{~g} / \mathrm{kg}$ of ICJ, ACJ, IWA and AWA yeasts.

Library preparation and sequencing. The sequencing was carried out on a Miseq platform following the Illumina $16 \mathrm{~S}$ metagenomic sequencing library preparation protocol (91). The cleaned PCR amplicons were multiplexed by dual indexing using the Nextera Index Kit v2 Set A (Illumina, California, USA, Cat. No. FC-131-2001). The index PCR products were cleaned using the AMPure beads and quantified using the Invitrogen ${ }^{\mathrm{TM}}$ Quant-iT ${ }^{\mathrm{TM}}$ Qubit $^{\mathrm{TM}}$ dsDNA BR (Broad range) assay kit (Thermo Fisher Scientific, California, USA, Cat. No. Q32853) with the Qubit 4 Fluorometer (Invitrogen ${ }^{\mathrm{TM}}$ ). To determine the library size representative, cleaned libraries were selected and analyzed using the Agilent DNA 1000 Kit (Agilent Technologies, California, USA, Cat. No. 067-1505). The libraries were diluted to 4 $\mathrm{nM}$ in $10 \mathrm{mM}$ Tris $(\mathrm{pH} 8.5)$ and pooled in an equal volume. The blank control samples with library concentrations lower than $4 \mathrm{nM}$ were pooled directly without further dilution. The pooled library was denatured using $0.2 \mathrm{~N} \mathrm{NaOH}$. Due to low diversity of the amplicon library, 5\% Illumina generated PhiX control (Illumina, San Diego, Waltham, MA, USA, Cat No: FC-110-3001) was spiked in by combining $570 \mu \mathrm{L}$ amplicon library with $30 \mu \mathrm{L}$ PhiX control. The library was then loaded at $8 \mathrm{pM}$ and sequenced on the Miseq System (Illumina, San Diego, California, USA) using the Miseq Reagent Kit v3 (600-cycle) (Illumina; catalog no., MS-102-3003). The sequencing was done in two runs. To prevent potential batch 
effects between sequencing runs, the digesta and the feed samples were distributed between the runs with consideration that each dietary treatment and each experimental tank were equally represented. Also, water and control samples were evenly distributed between the two runs.

Sequence data processing. The sequence data were processed in $\mathrm{R}$ (version 4.0.5) (92). For each sequencing run, DADA2 was used to process the raw sequence data and generate amplicon sequence variants (ASVs) (93). Briefly, the demultiplexed pair-ended reads were trimmed off the primer sequences (first $17 \mathrm{bps}$ of forward reads and first $21 \mathrm{bps}$ of reverse reads), truncated at the position where the median Phred quality score crashed (forward reads at position 300 bp and reverse reads at position $230 \mathrm{bp}$ for both runs) and filtered off low quality reads. After the trimming and filtering, a model of error rates was developed to remove error sequences. The forward and reverse reads were merged, and the ASV table for each run was constructed. The ASV table for each run were merged, and assigned with taxonomy using the reference database, SILVA version $138.1(94,95)$. A phyloseq object was constructed from the generated ASV table, the taxonomy table and the sample metadata using the phyloseq R package (version 1.34.0) (96). Taxa identified as chloroplasts or mitochondria were removed from the ASV table. The ASVs that had no phylum-level taxonomic assignments or appeared in less than three biological samples were conservatively filtered from the ASV table. The contaminating ASVs due to reagent contamination and cross contamination were identified and removed from ASV table as described elsewhere (30). The ASVs were then clustered using VSEARCH algorithm and subsequently curated with LULU (97). The post-clustering ASV table and representative sequences were used for the downstream data analysis. The core ASVs and alpha-diversity indices (observed ASVs, Pielou's evenness, Shannon's index and Faith's phylogenetic diversity (PD)) were computed according to Li et al. (30). Similarly, the beta-diversity indices (Jaccard distance, unweighted UniFrac distance, Aitchison distance and PhILR transformed Euclidean distance) were computed following Li et al. (30). The Jaccard distance and unweighted UniFrac distance were calculated by rarefying the ASV table into minimum sequence size i.e., 1,604 reads per sample (Fig. S9). Conversely, Aitchison distance and PhILR transformed Euclidean distance were computed using the unrarefied ASV table.

Metabolic reaction analysis of gut microbiota. The metabolic reaction analysis of gut microbiota was performed according to the method described by Yilmaz et al. (98). The ASVs for the digesta samples were mapped to metabolic reactions using an available collection of genomescale metabolic models (GSMMs) of gut microbes (99). Only ASVs that could be mapped to family or lower taxonomic rank and to at least one GSMM were included in the reaction level analysis. For each sample, we calculated the normalized abundance of each reaction based on equation (1):

$$
a_{\mathrm{r}}(i)=\frac{\sum_{j=1}^{n} a_{\mathrm{ASV}}(j) E(i, j)}{\sum_{j=1}^{n} a_{\mathrm{ASV}}(j)}
$$

where $a_{\mathrm{ASV}}(j)$ is the abundance of $\mathrm{ASV} j$ in the sample, $n$ is the total number of ASVs, and $\mathrm{E}(i, j)$ is the expected probability (frequency of occurrences) of reaction $i$ in the GSMMs mapped to $\mathrm{ASV} j$.

Statistical analysis. The statistical difference among the dietary groups for the microbial compositions at genus or lowest taxonomy ranks (top 15 most abundant taxa) were evaluated using Kruskal-Wallis test, followed by multiple comparison using Wilcox pair-wise comparison test. Similarly, the alpha-diversity measurements were evaluated using Kruskal-Wallis test and statistical differences among the dietary groups were detected using Wilcox pair-wise comparison test. The statistical difference among the dietary groups for the beta-diversity indices were computed using permutation multivariate analysis of variance (PERMANOVA) (100) with 999 permutations using the $\mathrm{R}$ package vegan 2.5.7 (101), followed by a pair-wise comparison. Principal coordinates analysis (PCoA) was used to visualize the betadiversity indices. The homogeneity of multivariate dispersions among the dietary groups was computed by permutation test, PERMDISP (102), using the R package vegan (101) and visually assessed with boxplots. Significant differences with adjusted $\mathrm{p}<0.05$ among dietary groups were detected using the Benjamini-Hochberg procedure (103). For the metabolic reaction analysis, mean abundance of each reaction was tested using a two-sample $t$-test for each pair of diets. Multiple testing was corrected using the BenjaminiHochberg procedure (103) and reactions with adjusted $\mathrm{p} \leq$ 0.05 were considered to be significantly different between diets. For each pair of diets, the enriched pathways among the significantly different reactions were computed using Fisher's exact test. The pathways with adjusted $\mathrm{p} \leq 0.05$ based on Benjamini-Hochberg procedure were considered to be enriched. Additionally, principal component analysis (PCA) was performed separately on standardized ASVs (Fig. S6) and reaction abundances (z-scores) (Fig. S7).

\section{Abbreviations}

ASVs: amplicon sequence variants; CJ: Cyberlindnera jadinii; ICJ: inactivated CJ; ACJ: autolyzed CJ; WA: Wickerhamomyces anomalus; IWA: inactivated WA; AWA: autolyzed WA; BR: broad range; FM: fishmeal; GSMMs: genome-scale metabolic models; HS: high sensitivity; LAB: lactic acid bacteria; MOS: mannan oligosaccharides; PCA: principal component analysis; PCoA: principal coordinates analysis; PD: phylogenetic diversity; PERMANOVA: permutation multivariate analysis of variance; SBM: soybean meal; SBMIE: SBM-induced enteritis; SPC: soy protein concentrates. 


\section{Declarations}

Ethics approval and consent to participate. The fish experiment was conducted at the research facility of Norwegian Institute of Water Resources (NIVA, Solbergstrand, Norway), which is a research facility approved by Norwegian Animal Research Authority (permit no. 174). The experimental procedures were in accordance with the national guidelines for the care and use of animals (The Norwegian Animal Welfare Act and the Norwegian Regulation on Animal experimentation).

Consent for publication. Not applicable

\section{Data and code availability}

The raw 16S rRNA gene sequence data and metadata files are deposited at the NCBI SRA database under the BioProject PRJNA797563. Other data and the code for reproducing the results are available in the Github repository (https://github.com/Jeleel2020/Salmon_ Yeasts_Microbiota).

\section{Competing interests}

The authors declared no competing interests

\section{Funding}

The current experiment was supported by Foods of Norway, a Centre for Research-based Innovation (the Research Council of Norway; grant no. 237841/030). The funding body had no role in the design of the study and collection, analysis, and interpretation of data, as well as in writing the manuscript.

\section{Author's contributions}

JOA, JØH, MØA, and MØ contributed to the conception, JOA, JØH, LTM and M $\varnothing$ designed the study. JOA, DDM, $\mathrm{J} \emptyset \mathrm{H}, \mathrm{SDCR}$ and DL involved in feed production, fish experiment and sampling. JOA and SDCR conducted the laboratory analysis. JOA and $O \varnothing$ performed the bioinformatics, statistical analyses, and data visualization. SJH, LTM and $\mathrm{M} \varnothing$ acquired funding. $\mathrm{J} \varnothing \mathrm{H}, \mathrm{M} \varnothing \mathrm{A}$ and $\mathrm{M} \varnothing$ supervised the work. JOA wrote the first draft of the manuscript. All the authors read, revised, and approved the final version of the manuscript for publication.

\section{Acknowledgments}

The authors are grateful to Ricardo Tavares Benicio for his technical support during the processing of the yeasts. We would like to appreciate Brankica Djordjevic, Anna Julie Tornes and the staff of the Fish laboratory at NIVA (Solbergstrand) for their help during the fish experiment. We are also grateful to Tan Thi Nguyen for her assistance with the MiSeq System.

\section{Reference}

1. Trine Ytrestøyl, Turid Synnøve Aas, and Torbjørn Åsgård. Utilisation of feed resources in production of atlantic salmon (salmo salar) in norway. Aquaculture, 448:365-374, 2015.

2. Turid Synnøve Aas, Trine Ytrestøyl, and Torbjørn Åsgård. Utilization of feed resources in the production of atlantic salmon (Salmo salar) in norway: An update for 2016. Aquaculture Reports, 15:100216, 2019.

3. Åshild Krogdahl, Michael Penn, Jim Thorsen, Ståle Refstie, and Anne Marie Bakke. Important antinutrients in plant feedstuffs for aquaculture: an update on recent findings regarding responses in salmonids. Aquaculture research, 41(3):333-344, 2010.

4. Åshild Krogdahl, Trond M Kortner, Alexander Jaramillo-Torres, Amr Ahmed Abdelrahim Gamil, Elvis Chikwati, Yanxian Li, Monica Schmidt, Eliot Herman, Theodore Hymowitz, Sepehr Teimouri, et al. Removal of three proteinaceous antinutrients from soybean does not mitigate soybean-induced enteritis in atlantic salmon (Salmo salar, L). Aquaculture, 514:734495, 2020

5. G Baeverfjord and Å Krogdahl. Development and regression of soybean meal induced enteritis in atlantic salmon, Salmo salar, L., distal intestine: a comparison with the intestines of fasted fish. Journal of Fish Diseases, 19(5):375-387, 1996.

6. Trond M Kortner, Stanko Skugor, Michael H Penn, Liv Torunn Mydland, Brankica Djordjevic, Marie Hillestad, Aleksei Krasnov, and Åshild Krogdahl. Dietary soyasaponin supplementation to pea protein concentrate reveals nutrigenomic interactions underlying enteropathy in atlantic salmon (Salmo salar). BMC veterinary research, 8(1):1-17, 2012.

7. PA Urán, JW Schrama, JHWM Rombout, A Obach, L Jensen, W Koppe, and JAJ Verreth. Soybean meal-induced enteritis in atlantic salmon (salmo salar I.) at different temperatures. Aquaculture Nutrition, 14(4):324-330, 2008.

8. TS Van Den Ingh and A Krogdahl. Negative effects of anti-nutritional factors from soybeans in salmonidae. Tijdschrift voor Diergeneeskunde, 115(20):935-938, 1990.

9. TSGAM Van den Ingh, Å Krogdahl, JJ Olli, HGCJM Hendriks, and JGJF Koninkx. Effects of soybean-containing diets on the proximal and distal intestine in atlantic salmon (salmo salar): a morphological study. Aquaculture, 94(4):297-305, 1991.

10. TSGAM Van den Ingh, JJ Olli, and Å Krogdahl. Alcohol-soluble components in soybeans cause morphological changes in the distal intestine of atlantic salmon, salmo salar I. Journal of Fish Diseases, 19(1):47-53, 1996.

11. Jillian P Fry, David C Love, Graham K MacDonald, Paul C West, Peder M Engstrom, Keeve E Nachman, and Robert S Lawrence. Environmental health impacts of feeding crops to farmed fish. Environment international, 91:201-214, 2016.

12. Markus Pahlow, PR Van Oel, MM Mekonnen, and Arjen Ysbert Hoekstra. Increasing pressure on freshwater resources due to terrestrial feed ingredients for aquaculture production. Science of the Total Environment, 536:847-857, 2015.

13. David Lapeña, Pernille M Olsen, Magnus $\varnothing$ Arntzen, Gergely Kosa, Volkmar Passoth, Vincent GH Eijsink, and Svein J Horn. Spruce sugars and poultry hydrolysate as growth medium in repeated fed-batch fermentation processes for production of yeast biomass. Bioprocess and biosystems engineering, 43(4):723-736, 2020.

14. Jeleel Opeyemi Agboola, Margareth Øverland, Anders Skrede, and Jon Øvrum Hansen. Yeast as major protein-rich ingredient in aquafeeds: a review of the implications for aquaculture production. Reviews in Aquaculture, 13(2):949-970, 2021.

15. Brett D Glencross, David Huyben, and Johan W Schrama. The application of single-cell ingredients in aquaculture feeds-a review. Fishes, 5(3):22, 2020.

16. Katheline Hua, Jennifer M Cobcroft, Andrew Cole, Kelly Condon, Dean R Jerry, Arnold Mangott, Christina Praeger, Matthew J Vucko, Chaoshu Zeng, and Kyall Zenger. The future of aquatic protein: implications for protein sources in aquaculture diets. One Earth, 1(3):316-329, 2019

17. Jessica L Couture, Roland Geyer, Jon Øvrum Hansen, Brandon Kuczenski, Margareth Øverland, Joseph Palazzo, Christian Sahlmann, and Hunter Lenihan. Environmental benefits of novel nonhuman food inputs to salmon feeds. Environmental science \& technology, 53(4):1967-1975, 2019.

18. Jeleel Opeyemi Agboola, Marion Schiavone, Margareth Øverland, Byron Morales-Lange, Leidy Lagos, Magnus Øverlie Arntzen, David Lapeña, Vincent GH Eijsink, Svein Jarle Horn, Liv Torunn Mydland, et al. Impact of down-stream processing on functional properties of yeasts and the implications on gut health of atlantic salmon (salmo salar). Scientific reports, 11(1):1-14, 2021.

19. Fabian Grammes, Felipe Eduardo Reveco, Odd Helge Romarheim, Thor Landsverk, Liv Torunn Mydland, and Margareth Øverland. Candida utilis and chlorella vulgaris counteract intestinal inflammation in atlantic salmon (salmo salar I.). PloS one, 8(12):e83213, 2013.

20. Qinghui Ai, Houguo Xu, Kangsen Mai, Wei Xu, Jun Wang, and Wenbing Zhang. Effects of dietary supplementation of bacillus subtilis and fructooligosaccharide on growth performance, survival, non-specific immune response and disease resistance of juvenile large yellow croaker, larimichthys crocea. Aquaculture, 317(1-4):155-161, 2011.

21. Andrew Bell and Nathalie Juge. Mucosal glycan degradation of the host by the gut microbiota. Glycobiology, 31(6):691-696, 2021.

22. Clara Belzer. Nutritional strategies for mucosal health: The interplay between microbes and mucin glycans. Trends in microbiology, 2021.

23. Karina Gajardo, Alexander Jaramillo-Torres, Trond M Kortner, Daniel L Merrifield, John Tinsley, Anne Marie Bakke, and Åshild Krogdahl. Alternative protein sources in the diet modulate microbiota and functionality in the distal intestine of atlantic salmon (salmo salar). Applied and environmental microbiology, 83(5):e02615-16, 2017.

24. Felipe E Reveco, Margareth Øverland, Odd H Romarheim, and Liv T Mydland. Intestinal bacterial community structure differs between healthy and inflamed intestines in atlantic salmon (salmo salar I.). Aquaculture, 420:262-269, 2014.

25. Victor Schmidt, Linda Amaral-Zettler, John Davidson, Steven Summerfelt, and Christopher Good. Influence of fishmeal-free diets on microbial communities in atlantic salmon (salmo salar) recirculation aquaculture systems. Applied and Environmental Microbiology, 82(15): 4470-4481, 2016.

26. P Navarrete, P Fuentes, L De la Fuente, L Barros, F Magne, R Opazo, C Ibacache, R Espejo, and J Romero. Short-term effects of dietary soybean meal and lactic acid bacteria on 
the intestinal morphology and microbiota of a tlantic salmon (s almo salar). Aquaculture Nutrition, 19(5):827-836, 2013.

27. Gajardo K, Rodiles A, Kortner TM, Krogdahl Å, Bakke AM, Merrifield DL, and Sørum H. A high-resolution map of the gut microbiota in atlantic salmon (salmo salar): a basis for comparative gut microbial research. Scientific Reports, 6:1-10, 2016.

28. Colin Fogarty, Catherine M Burgess, Paul D Cotter, Raul Cabrera-Rubio, Paul Whyte, Conor Smyth, and Declan J Bolton. Diversity and composition of the gut microbiota of atlantic salmon (salmo salar) farmed in irish waters. Journal of applied microbiology, 127 (3):648-657, 2019.

29. Carola E Dehler, Christopher J Secombes, and Samuel AM Martin. Seawater transfer alters the intestinal microbiota profiles of atlantic salmon (salmo salar I.). Scientific reports, $7(1): 1-11,2017$.

30. Yanxian Li, Leonardo Bruni, Alexander Jaramillo-Torres, Karina Gajardo, Trond M Kortner and Åshild Krogdahl. Differential response of digesta-and mucosa-associated intestinal microbiota to dietary insect meal during the seawater phase of atlantic salmon. Animal microbiome, 3(1):1-18, 2021.

31. Einar Ringø and François-Joël Gatesoupe. Lactic acid bacteria in fish: a review. Aquaculture, 160(3-4):177-203, 1998

32. Einar Ringø, Seyed Hossein Hoseinifar, Koushik Ghosh, Hien Van Doan, Bo Ram Beck, and Seong Kyu Song. Lactic acid bacteria in finfish—an update. Frontiers in Microbiology, page 1818,2018

33. Daniel L Merrifield, José Luis Balcázar, Carly Daniels, Zhigang Zhou, Oliana Carnevali, Yun-Zhang Sun, Seyed Hossein Hoseinifar, and Einar Ringø. Indigenous lactic acid bacteria in fish and crustaceans. Aquaculture nutrition: gut health, probiotics and prebiotics, pages 128-168, 2014

34. Mirco Vacca, Giuseppe Celano, Francesco Maria Calabrese, Piero Portincasa, Marco Gobbetti, and Maria De Angelis. The controversial role of human gut lachnospiraceae. Microorganisms, 8(4):573, 2020.

35. Guangming Ren, Liming $\mathrm{Xu}$, Tongyan Lu, Yongquan Zhang, Yuanyuan Wang, and Jiasheng Yin. Protective effects of lentinan on lipopolysaccharide induced inflammatory response in intestine of juvenile taimen (hucho taimen, pallas). International journal of biological macromolecules, 121:317-325, 2019.

36. Yang Jin, Inga Leena Angell, Simen Rød Sandve, Lars Gustav Snipen, Yngvar Olsen, and Knut Rudi. Atlantic salmon raised with diets low in long-chain polyunsaturated n-3 fatty acids in freshwater have a mycoplasma-dominated gut microbiota at sea. Aquaculture Environment Interactions, 11:31-39, 2019

37. Shruti Gupta, Adriána Fečkaninová, Jep Lokesh, Jana Koščová, Mette Sørensen, Jorge Fernandes, and Viswanath Kiron. Lactobacillus dominate in the intestine of atlantic salmon fed dietary probiotics. Frontiers in microbiology, 9:3247, 2019.

38. Shruti Gupta, Jep Lokesh, Yousri Abdelhafiz, Prabhugouda Siriyappagouder, Ronan Pierre, Mette Sørensen, Jorge MO Fernandes, and Viswanath Kiron. Macroalga-derived alginate oligosaccharide alters intestinal bacteria of atlantic salmon. Frontiers in microbiology, page 2037, 2019

39. Martin S Llewellyn, Philip McGinnity, Melanie Dionne, Justine Letourneau, Florian Thonier, Gary R Carvalho, Simon Creer, and Nicolas Derome. The biogeography of the atlantic salmon (salmo salar) gut microbiome. The ISME journal, 10(5):1280-1284, 2016.

40. William E Holben, Paul Williams, M Saarinen, LK Särkilahti, and Juha HA Apajalahti. Phylogenetic analysis of intestinal microflora indicates a novel mycoplasma phylotype in farmed and wild salmon. Microbial ecology, 44(2):175-185, 2002.

41. Jie Wang, Alexander Jaramillo-Torres, Yanxian Li, Trond M Kortner, Karina Gajardo, Øyvind Jakobsen Brevik, Jan Vidar Jakobsen, and Åshild Krogdahl. Microbiota in intestina digesta of atlantic salmon (salmo salar), observed from late freshwater stage until one yea in seawater, and effects of functional ingredients: a case study from a commercial sized research site in the arctic region. Animal microbiome, 3(1):1-16, 2021.

42. E Ringø, TH Birkbeck, PO Munro, O Vadstein, and $\mathrm{K}$ Hjelmeland. The effect of early exposure to vibrio pelagius on the aerobic bacterial flora of turbot, scophthalmus maximus (I.) larvae. Journal of applied bacteriology, 81(2):207-211, 1996

43. GH Hansen, E Strøm, and JA Olafsen. Effect of different holding regimens on the intestina microflora of herring (clupea harengus) larvae. Applied and Environmental Microbiology, 58(2):461-470, 1992

44. Atul R Desai, Matthew G Links, Stephanie A Collins, Graeme S Mansfield, Murray D Drew, Andrew G Van Kessel, and Janet E Hill. Effects of plant-based diets on the distal gut microbiome of rainbow trout (oncorhynchus mykiss). Aquaculture, 350:134-142, 2012.

45. Timothy J Green, Richard Smullen, and Andrew C Barnes. Dietary soybean protein concentrate-induced intestinal disorder in marine farmed atlantic salmon, salmo salar is associated with alterations in gut microbiota. Veterinary microbiology, 166(1-2):286-292, 2013

46. M Hartviksen, JLG Vecino, E Ringø, A-M Bakke, S Wadsworth, Å Krogdahl, K Ruohonen, and $A$ Kettunen. Alternative dietary protein sources for a tlantic salmon (s almo salar I.) effect on intestinal microbiota, intestinal and liver histology and growth. Aquaculture Nutrition, 20(4):381-398, 2014.

47. Jeleel O. Agboola, Dominic D. Mensah, Jon Ø. Hansen, David Lapeña, Liv T. Mydland Magnus Ø. Arntzen, Svein J. Horn, Ove Øyås, Charles McLean Press, and Margareth Øverland. Effects of yeast species and processing on intestinal health and transcriptomic profiles of atlantic salmon (salmo salar) fed soybean meal-based diets in seawater. International Journal of Molecular Sciences, 23(3), 2022.

48. David Huyben, Andreas Nyman, Aleksandar Vidaković, Volkmar Passoth, Richard Moccia Anders Kiessling, Johan Dicksved, and Torbjörn Lundh. Effects of dietary inclusion of the yeasts saccharomyces cerevisiae and wickerhamomyces anomalus on gut microbiota of rainbow trout. Aquaculture, 473:528-537, 2017.

49. David Huyben, Li Sun, Rich Moccia, Anders Kiessling, Johan Dicksved, and Thomas Lundh. Dietary live yeast and increased water temperature influence the gut microbiota of rainbow trout. Journal of Applied Microbiology, 124(6):1377-1392, 2018.

50. Arkadios Dimitroglou, Daniel Lee Merrifield, Peter Spring, John Sweetman, Roy Moate, and Simon John Davies. Effects of mannan oligosaccharide (mos) supplementation on growth performance, feed utilisation, intestinal histology and gut microbiota of gilthead sea bream (sparus aurata). Aquaculture, 300(1-4):182-188, 2010.

51. Silvia Torrecillas, Daniel Montero, and Marisol Izquierdo. Improved health and growth of fish fed mannan oligosaccharides: potential mode of action. Fish \& shellfish immunology, 36(2):525-544, 2014.

52. Huali Wang, Guijie Chen, Xiang Li, Fuping Zheng, and Xiaoxiong Zeng. Yeast $\beta$-glucan, a potential prebiotic, showed a similar probiotic activity to inulin. Food \& Function, 11(12): 10386-10396, 2020

53. Mengdai Xu, Xiaoxing Mo, Hao Huang, Xi Chen, Hongjie Liu, Zhao Peng, Liangkai Chen, Shuang Rong, Wei Yang, Shufang Xu, et al. Yeast $\beta$-glucan alleviates cognitive deficit by regulating gut microbiota and metabolites in a $\beta 1-42$-induced ad-like mice. International journal of biological macromolecules, 161:258-270, 2020

54. Jules Petit, Irene de Bruijn, Mark RG Goldman, Erik van den Brink, Wilbert F Pellikaan, Maria Forlenza, and Geert F Wiegertjes. $\beta$-glucan-induced immuno-modulation: A role for the intestinal microbiota and short-chain fatty acids in common carp. Frontiers in im munology, 12:761820, 2022.

55. S Rimoldi, E Gini, JFA Koch, F lannini, F Brambilla, and G Terova. Effects of hydrolyzed fish protein and autolyzed yeast as substitutes of fishmeal in the gilthead sea bream (sparus aurata) diet, on fish intestinal microbiome. BMC Veterinary Research, 16(1):1-13, 2020.

56. Charles MAP Franz, Akihito Endo, Hikmate Abriouel, Carol A Van Reenen, Antonio Gálvez, and Leon MT Dicks. The genus pediococcus. Lactic acid bacteria: biodiversity and taxonomy, pages 359-376, 2014

57. Radhey S Gupta, Sudip Patel, Navneet Saini, and Shu Chen. Robust demarcation of 17 distinct bacillus species clades, proposed as novel bacillaceae genera, by phylogenomics and comparative genomic analyses: description of robertmurraya kyonggiensis $\mathrm{sp}$. nov. and proposal for an emended genus bacillus limiting it only to the members of the subtilis and cereus clades of species. International Journal of Systematic and Evolutionary Microbiology, 70(11):5753-5798, 2020

58. Joanne B Emerson, Rachel I Adams, Clarisse M Betancourt Román, Brandon Brooks, David A Coil, Katherine Dahlhausen, Holly H Ganz, Erica M Hartmann, Tiffany Hsu, Nicholas B Justice, et al. Schrödinger's microbes: tools for distinguishing the living from the dead in microbial ecosystems. Microbiome, 5(1):1-23, 2017.

59. Jon Øvrum Hansen, Leidy Lagos, Peng Lei, Felipe Eduardo Reveco-Urzua, Byron Morales-Lange, Line Degn Hansen, Marion Schiavone, Liv Torunn Mydland, Magnus Øverlie Arntzen, Luis Mercado, et al. Down-stream processing of baker's yeast (saccharomyces cerevisiae)-effect on nutrient digestibility and immune response in atlantic salmon (salmo salar). Aquaculture, 530:735707, 2021

60. Marion Schiavone, Nathalie Sieczkowski, Mathieu Castex, Emmanuelle Trevisiol, Etienne Dague, and Jean Marie François. Afm dendritips functionalized with molecular probes specific to cell wall polysaccharides as a tool to investigate cell surface structure and organization. The Cell Surface, 5:100027, 2019.

61. Anusha KS Dhanasiri, Alexander Jaramillo Torres, Elvis M Chikwati, Torunn Forberg, Åshild Krogdahl, and Trond M Kortner. Effects of dietary supplementation with prebiotic and pediococcus acidilactici on gut health, transcriptome, microbiome, and metabolome in atlantic salmon (salmo salar I) after seawater transfer. Research sqaure(pre-print.

62. Alexander Jaramillo-Torres, Mark D Rawling, Ana Rodiles, Heidi E Mikalsen, Lill-Heidi Johansen, John Tinsley, Torunn Forberg, Elisabeth Aasum, Mathieu Castex, and Daniel Lee Merrifield. Influence of dietary supplementation of probiotic pediococcus acidilactic ma18/5m during the transition from freshwater to seawater on intestinal health and $\mathrm{mi}$ crobiota of atlantic salmon (salmo salar I.). Frontiers in microbiology, page 2243, 2019.

63. A Abid, SJ Davies, P Waines, M Emery, M Castex, G Gioacchini, O Carnevali, R Bickerdike, J Romero, and DL Merrifield. Dietary synbiotic application modulates atlantic salmon (salmo salar) intestinal microbial communities and intestinal immunity. Fish \& shellfish immunology, 35(6):1948-1956, 2013.

64. Aweeda Newaj-Fyzul, Abiodun A Adesiyun, A Mutani, A Ramsubhag, Jason Brunt, an Brian Austin. Bacillus subtilis ab1 controls aeromonas infection in rainbow trout (oncorhynchus mykiss, walbaum). Journal of applied microbiology, 103(5):1699-1706, 2007.

65. Milica Ciric, David Waite, Jenny Draper, and John Brian Jones. Characterization of mid-intestinal microbiota of farmed chinook salmon using $16 \mathrm{~s}$ rrna gene metabarcoding. Archives of Biological Sciences, 71(4):577-587, 2019.

66. Xingkun Jin, Ziwei Chen, Yan Shi, Jian-Fang Gui, and Zhe Zhao. Response of gut microbiota to feed-borne bacteria depends on fish growth rate: a snapshot survey of farmed juvenile takifugu obscurus. Microbial Biotechnology, 2021.

67. Jeremiah J Minich, Barbara Nowak, Abigail Elizur, Rob Knight, Stewart Fielder, and Eric Allen. Impacts of the marine hatchery built environment, water and feed on mucosal microbiome colonization across ontogeny in yellowtail kingfish, seriola lalandi. Frontiers in Marine Science, 8:516, 2021.

68. Jackson Wilkes Walburn, Bernd Wemheuer, Torsten Thomas, Elizabeth Copeland, Wayn O'Connor, Mark Booth, Stewart Fielder, and Suhelen Egan. Diet and diet-associated bacteria shape early microbiome development in yellowtail kingfish (seriola lalandi). Microbia biotechnology, 12(2):275-288, 2019.

69. Sandi Wong, W Zac Stephens, Adam R Burns, Keaton Stagaman, Lawrence A David, Brendan JM Bohannan, Karen Guillemin, and John F Rawls. Ontogenetic differences in dietary fat influence microbiota assembly in the zebrafish gut. MBio, 6(5):e00687-15, 2015.

70. Yanxian Li, Karina Gajardo, Alexander Jaramillo-Torres, Trond M Kortner, and Åshild Krog dahl. Consistent changes in the intestinal microbiota of atlantic salmon fed insect mea diets. Animal microbiome, 4(1):1-15, 2022.

71. Jeremiah J Minich, Greg D Poore, Khattapan Jantawongsri, Colin Johnston, Kate Bowie, John Bowman, Rob Knight, Barbara Nowak, and Eric E Allen. Microbial ecology of at antic salmon (salmo salar) hatcheries: impacts of the built environment on fish mucosa microbiota. Applied and environmental microbiology, 86(12):e00411-20, 2020.

72. Christos Giatsis, Detmer Sipkema, Hauke Smidt, Hans Heilig, Giulia Benvenuti, Johan Verreth, and Marc Verdegem. The impact of rearing environment on the development of gut microbiota in tilapia larvae. Scientific reports, 5(1):1-15, 2015.

73. Tamsyn M Uren Webster, Sofia Consuegra, Matthew Hitchings, and Carlos Garcia de Leaniz. Interpopulation variation in the atlantic salmon microbiome reflects environmental 
and genetic diversity. Applied and Environmental Microbiology, 84(16):e00691-18, 2018.

74. XM Li, YJ Zhu, QY Yan, Einar Ringø, and DG Yang. Do the intestinal microbiotas differ between paddlefish ( $p$ olyodon spathala) and bighead carp (a ristichthys nobilis) reared in the same pond? Journal of applied microbiology, 117(5):1245-1252, 2014.

75. Pabodha Weththasinghe, Sérgio DC Rocha, Ove Øyås, Leidy Lagos, Jon Ø Hansen, Liv T Mydland, and Margareth Øverland. Modulation of atlantic salmon (salmo salar) gut microbiota composition and predicted metabolic capacity by feeding diets with processed black soldier fly (hermetia illucens) larvae meals and fractions. Animal microbiome, 4(1):1-21, 2022.

76. Aprajita Singh, Sajjad Karimi, Aleksandar Vidakovic, Johan Dicksved, Markus Langeland, Jorge A Ferreira, Mohammad J Taherzadeh, Anders Kiessling, and Torbjörn Lundh. Dietary filamentous fungi and duration of feeding modulates gut microbial composition in rainbow trout (oncorhynchus mykiss). Frontiers in Marine Science.

77. ML Usher, C Talbot, and FB Eddy. Effects of transfer to seawater on digestion and gut function in atlantic salmon smolts (salmo salar I.). Aquaculture, 90(1):85-96, 1990.

78. Kamarul Zaman Zarkasi, Richard S Taylor, Guy CJ Abell, Mark L Tamplin, Brett D Glencross, and John P Bowman. Atlantic salmon (salmo salar I.) gastrointestinal microbial community dynamics in relation to digesta properties and diet. Microbial ecology, 71(3): 589-603, 2016.

79. Lora V Hooper, Dan R Littman, and Andrew J Macpherson. Interactions between the microbiota and the immune system. science, 336(6086):1268-1273, 2012.

80. Ema Damayanti, Lies Mira Yusiati, and Achmad Dinoto. 16s rrna identification of pediococcus spp. from broiler and studies of adherence ability on immobilized mucus. Indonesian Journal of Biotechnology, 17(2):96-106, 2012.

81. Praveen P Balgir, Baljinder Kaur, Tejinder Kaur, Natisha Daroch, and Gurpreet Kaur. In vitro and in vivo survival and colonic adhesion of pediococcus acidilactici mtcc5101 in human gut. BioMed research international, 2013, 2013.

82. Kaitlyn Oliphant and Emma Allen-Vercoe. Macronutrient metabolism by the human gut microbiome: major fermentation by-products and their impact on host health. Microbiome, 7(1):1-15, 2019.

83. Renan Corrêa-Oliveira, José Luís Fachi, Aline Vieira, Fabio Takeo Sato, and Marco Aurélio $\mathrm{R}$ Vinolo. Regulation of immune cell function by short-chain fatty acids. Clinical \& translational immunology, 5(4):e73, 2016.

84. Julie AK McDonald, Benjamin H Mullish, Alexandros Pechlivanis, Zhigang Liu, Jerusa Brignardello, Dina Kao, Elaine Holmes, Jia V Li, Thomas B Clarke, Mark R Thursz, et al. Inhibiting growth of clostridioides difficile by restoring valerate, produced by the intestinal microbiota. Gastroenterology, 155(5):1495-1507, 2018.

85. Mazen Issa, Ashwin N Ananthakrishnan, and David G Binion. Clostridium difficile and inflammatory bowel disease. Inflammatory bowel diseases, 14(10):1432-1442, 2008.

86. PP Lyons, JF Turnbull, Karl A Dawson, and Margaret Crumlish. Phylogenetic and functional characterization of the distal intestinal microbiome of rainbow trout oncorhynchus mykiss from both farm and aquarium settings. Journal of Applied Microbiology, 122(2): 347-363, 2017.

87. Morgan GI Langille, Jesse Zaneveld, J Gregory Caporaso, Daniel McDonald, Dan Knights, Joshua A Reyes, Jose C Clemente, Deron E Burkepile, Rebecca L Vega Thurber, Rob Knight, et al. Predictive functional profiling of microbial communities using $16 \mathrm{~s}$ rrna marker gene sequences. Nature biotechnology, 31(9):814-821, 2013.

88. Shan Sun, Roshonda B Jones, and Anthony A Fodor. Inference-based accuracy of metagenome prediction tools varies across sample types and functional categories. $\mathrm{Mi}$ crobiome, 8(1):1-9, 2020

89. National Research Council. Nutrient requirements of fish and shrimp. 2011.

90. P Antony Jesu Prabhu, Erik-Jan Lock, Gro-Ingunn Hemre, Kristin Hamre, Marit Espe, Pål A Olsvik, Joana Silva, Ann-Cecilie Hansen, Johan Johansen, Nini H Sissener, et al. Recommendations for dietary level of micro-minerals and vitamin $\mathrm{d} 3 \mathrm{to}$ atlantic salmon (salmo salar) parr and post-smolt when fed low fish meal diets. PeerJ, 7:e6996, 2019.

91. Inc Illumina. 16s metagenomic sequencing library preparation. preparing $16 \mathrm{~s}$ ribosomal rna gene amplicons for the illumina miseq system. 16S Metagenomic Sequencing Library Preparation Manual, pages 1-23.

92. R Core Team et al. R: A language and environment for statistical computing. 2013.

93. Benjamin J Callahan, Paul J McMurdie, Michael J Rosen, Andrew W Han, Amy Jo A Johnson, and Susan $P$ Holmes. Dada2: High-resolution sample inference from illumina amplicon data. Nature methods, 13(7):581-583, 2016.

94. Christian Quast, Elmar Pruesse, Pelin Yilmaz, Jan Gerken, Timmy Schweer, Pablo Yarza, Jörg Peplies, and Frank Oliver Glöckner. The silva ribosomal rna gene database project: improved data processing and web-based tools. Nucleic acids research, 41(D1):D590D596, 2012.

95. Pelin Yilmaz, Laura Wegener Parfrey, Pablo Yarza, Jan Gerken, Elmar Pruesse, Christian Quast, Timmy Schweer, Jörg Peplies, Wolfgang Ludwig, and Frank Oliver Glöckner. The silva and "all-species living tree project (Itp)" taxonomic frameworks. Nucleic acids research, 42(D1):D643-D648, 2014.

96. Paul J McMurdie and Susan Holmes. phyloseq: an r package for reproducible interactive analysis and graphics of microbiome census data. PloS one, 8(4):e61217, 2013.

97. Tobias Guldberg Frøslev, Rasmus Kjøller, Hans Henrik Bruun, Rasmus Ejrnæs, Ane Kirstine Brunbjerg, Carlotta Pietroni, and Anders Johannes Hansen. Algorithm for postclustering curation of dna amplicon data yields reliable biodiversity estimates. Nature communications, 8(1):1-11, 2017.

98. Bahtiyar Yilmaz, Pascal Juillerat, Ove Øyås, Charlotte Ramon, Francisco Damian Bravo, Yannick Franc, Nicolas Fournier, Pierre Michetti, Christoph Mueller, Markus Geuking, Valerie E. H. Pittet, Michel H. Maillard, Gerhard Rogler, Swiss IBD Cohort Investigators, Reiner Wiest, Jörg Stelling, and Andrew J. Macpherson. Microbial network disturbances in relapsing refractory crohn's disease. Nature Medicine, 25:323-336, 2019.

99. Magnúsdóttir S, Heinken A, Kutt L, Ravcheev DA, Bauer E, Noronha A, Greenhalgh K, Jäger C, Baginska J, and Wilmes P. Generation of genome-scale metabolic reconstructions for 773 members of the human gut microbiota. Nature Biotechnology, 35:81-89, 2017.

100. Anderson MJ. A new method for non-parametric multivariate analysis of variance. Austral
Ecology, 26:32-46, 2001.

101. Oksanen J, Blanchet F, Friendly M, Kindt R, Legendre P, McGlinn D, Minchin P, O'Hara R, Simpson G, and Solymos P. vegan: community ecology package. 2019.

102. Anderson MJ. Distance-based tests for homogeneity of multivariate dispersions. Biometrics, 62:245-253, 2006

103. Benjamini $Y$ and Hochberg $Y$. Controlling the false discovery rate: a practical and powerful approach to multiple testing. Journal of Royal Statistical Society: Series B (Methodlogy), 62:245-253, 2006. 
Supplementary Note 1: Supplemental tables

Table S1. The dominant taxa identified as contaminants in the negative controls and the blank filter papers. (available as additional file 2_supplementary tables in the GitHub repository: https://github.com/Jeleel2020/Salmon_Yeasts_Microbiota/ tree/main/Results

Table S2. The prevalence of core ASVs in the digesta of fish fed the experimental diets. (available as additional file 2_supplementary tables in the GitHub repository: https://github.com/Jeleel2020/Salmon_Yeasts_Microbiota/tree/main/Results 
Table S3. Pair-wise comparisons of alpha-diversity indices of gut microbiota in Atlantic salmon smolts fed FM-based diet or SBM-based diet with yeasts. ${ }^{1}$

\begin{tabular}{lcccc}
\hline & Observed ASVs & Pielou's evenness & Shannon's index & Faith's PD \\
\hline P-values $^{2}$ & $<0.0001$ & $<0.0001$ & $<0.0001$ & $<0.0001$ \\
Pair-wise comparisons & & & & \\
IWAvsAWA & $<0.0001$ & $<0.0001$ & $<0.0001$ & $<0.0001$ \\
IWAvsACJ & 0.003 & $<0.0001$ & $<0.0001$ & 0.001 \\
IWAvsSBM & 0.81 & 1.000 & 0.9701 & 0.740 \\
IWAvsICJ & 0.77 & 1.000 & 0.9701 & 0.740 \\
IWAvsFM & 0.001 & 1.000 & $<0.0001$ & $<0.0001$ \\
AWAvsACJ & 0.62 & 0.052 & 0.007 & 0.580 \\
AWAvsSBM & $<0.0001$ & $<0.0001$ & $<0.0001$ & $<0.0001$ \\
AWAvsICJ & $<0.0001$ & $<0.0001$ & $<0.0001$ & $<0.0001$ \\
AWAvsFM & $<0.0001$ & $<0.0001$ & $<0.0001$ & $<0.0001$ \\
ACJvsSBM & 0.010 & $<0.0001$ & $<0.0001$ & 0.003 \\
ACJvsICJ & 0.010 & $<0.0001$ & $<0.0001$ & 0.002 \\
ACJvsFM & $<0.0001$ & $<0.0001$ & $<0.0001$ & $<0.0001$ \\
SBMvsICJ & 0.620 & 1.000 & 0.970 & 0.500 \\
SBMvsFM & 0.002 & 1.000 & 0.001 & 0.001 \\
ICJvsFM & $<0.0001$ & 0.910 & $<0.0001$ & $<0.0001$
\end{tabular}

${ }^{1}$ The diets are: FM - fishmeal-based; SBM - soybean meal-based; 4 other diets containing $300 \mathrm{~g} / \mathrm{kg} \mathrm{SBM}$ and $100 \mathrm{~g} / \mathrm{kg}$ of ICJ - inactivated Cyberlindnera jadinii; ACJ - autolyzed C. jadinii; IWA - inactivated Wickerhamomyces anomalus; AWA autolyzed $W$. anomalus diets.

${ }^{2} \mathrm{P}$-values computed for diet effect with Kruskal-Wallis test.

${ }^{3}$ Wilcox pairwise comparison to identify differences between diets. 
Table S4. PERMANOVA analysis for beta-diversity of gut microbiota in Atlantic salmon smolts fed FM-based diet or SBM-based diet with yeasts. ${ }^{1}$

\begin{tabular}{|c|c|c|c|c|}
\hline & Jaccard dist. $^{2}$ & Unw. Unifrac dist. $^{2}$ & Aitchison dist. $^{3}$ & PhILR dist. ${ }^{3}$ \\
\hline P-values ${ }^{4}$ & $<0.001$ & $<0.001$ & $<0.001$ & $<0.001$ \\
\hline \multicolumn{5}{|c|}{ Pair-wise comparisons ${ }^{3}$} \\
\hline IWAvsAWA & 0.015 & 0.015 & 0.015 & 0.015 \\
\hline IWAvsACJ & 0.015 & 0.015 & 0.015 & 0.015 \\
\hline IWAvsSBM & 0.600 & 0.27 & 0.075 & 0.015 \\
\hline IWAvsICJ & 1.000 & 1.000 & 1.000 & 0.345 \\
\hline IWAvsFM & 0.015 & 0.015 & 0.015 & 0.015 \\
\hline AWAvsACJ & 0.015 & 0.015 & 0.015 & 0.015 \\
\hline AWAvsSBM & 0.015 & 0.015 & 0.015 & 0.015 \\
\hline AWAvsICJ & 0.015 & 0.015 & 0.015 & 0.015 \\
\hline AWAvsFM & 0.015 & 0.015 & 0.015 & 0.015 \\
\hline ACJvsSBM & 0.015 & 0.015 & 0.015 & 0.015 \\
\hline ACJvsICJ & 0.015 & 0.015 & 0.015 & 0.015 \\
\hline ACJvsFM & 0.015 & 0.015 & 0.015 & 0.015 \\
\hline SBMvsICJ & 0.600 & 1.000 & 0.195 & 1.000 \\
\hline SBMvsFM & 0.015 & 0.015 & 0.015 & 0.015 \\
\hline ICJvsFM & 0.015 & 0.015 & 0.015 & 0.015 \\
\hline
\end{tabular}

${ }^{1}$ The diets are: FM - fishmeal-based; SBM - soybean meal-based; 4 other diets containing $300 \mathrm{~g} / \mathrm{kg}$ SBM and $100 \mathrm{~g} / \mathrm{kg}$ of ICJ - inactivated Cyberlindnera jadinii; ACJ - autolyzed C. jadinii; IWA - inactivated Wickerhamomyces anomalus; AWA autolyzed W. anomalus diets. Jaccard dist. - Jaccard distance; Unw. Unifrac dist. - Unweighted Unifrac distance; Aitchison dist. - Robust Aitchison distance; and PhILR dist. - Phylogenetic isometric log-ratio (PhILR) transformed Euclidean distance ${ }^{2}$ Performed on phyloseq object rarefied to minimum read sequence in the sample.

${ }^{3}$ Performed on unrarefied phyloseq object.

${ }^{4} \mathrm{P}$-values of permutational multivariate analysis of variance (PERMANOVA) test for the four beta-diversity distances.

${ }^{5}$ PERMANOVA pairwise comparisons for the four beta-diversity distances. 
Table S5. Test of homogeneity of multivariate dispersions among dietary groups.

\begin{tabular}{lcccc}
\hline & Jaccard dist. $^{2}$ & Unw. Unifrac dist. $^{2}$ & Aitchison dist. $^{3}$ & PhILR dist. $^{3}$ \\
\hline P-values & 0.001 & 0.001 & 0.001 & 0.002 \\
Pair-wise comparisons & & & \\
IWAvsAWA & $<0.001$ & 0.023 & $<0.001$ & 0.018 \\
IWAvsACJ & $<0.001$ & 0.032 & $<0.001$ & 0.824 \\
IWAvsSBM & 0.412 & 0.195 & 0.039 & 0.668 \\
IWAvsICJ & 0.84 & 0.90 & 0.109 & 0.648 \\
IWAvsFM & 0.051 & 0.009 & 0.041 & 0.150 \\
AWAvsACJ & 0.328 & 0.80 & 0.017 & 0.028 \\
AWAvsSBM & 0.001 & 0.002 & 0.001 & 0.002 \\
AWAvsICJ & 0.001 & 0.026 & 0.001 & 0.001 \\
AWAvsFM & 0.001 & 0.001 & 0.001 & 0.857 \\
ACJvsSBM & 0.007 & 0.002 & 0.001 & 0.522 \\
ACJvsICJ & 0.058 & 0.041 & 0.001 & 0.508 \\
ACJvsFM & 0.001 & 0.001 & 0.001 & 0.205 \\
SBMvsICJ & 0.418 & 0.24 & 0.471 & 0.950 \\
SBMvsFM & 0.333 & 0.214 & 0.842 & 0.085 \\
ICJvsFM & 0.086 & 0.013 & 0.402 & 0.083 \\
\hline
\end{tabular}

${ }^{1}$ The diets are: FM - fishmeal-based; SBM - soybean meal-based; 4 other diets containing $300 \mathrm{~g} / \mathrm{kg} \mathrm{SBM}$ and $100 \mathrm{~g} / \mathrm{kg}$ of ICJ - inactivated Cyberlindnera jadinii; ACJ - autolyzed C. jadinii; IWA - inactivated Wickerhamomyces anomalus; AWA autolyzed W. anomalus diets. Jaccard dist. - Jaccard distance; Unw. Unifrac dist. - Unweighted Unifrac distance; Aitchison dist. - Robust Aitchison distance; and PhILR dist. - Phylogenetic isometric log-ratio (PhILR) transformed Euclidean distance ${ }^{2}$ Performed on phyloseq object rarefied to minimum read sequence in the sample.

${ }^{3}$ Performed on unrarefied phyloseq object.

${ }^{4} \mathrm{P}$-values of homogeneity of multivariate dispersions using PERMDISP test for the four beta-diversity distances.

${ }^{5}$ PERMDISP pairwise comparisons for the four beta-diversity distances. 
Table S6. Composition of spray-dried yeasts with and without the autolysis treatment. All values are presented in \% DM, except gross energy which is presented as $\mathrm{MJ} / \mathrm{kg} \mathrm{DM}$.

\begin{tabular}{lcccc}
\hline & \multicolumn{2}{c}{ Cyberlindnera jadinii } & \multicolumn{2}{c}{ Wickerhamomyces anomalus } \\
\hline & Inactivated & Autolyzed & Inactivated & Autolyzed \\
\hline DM (\%) & $96.3 \pm 0.03$ & $93.1 \pm 0.04$ & $96.1 \pm 0.02$ & $96.1 \pm 0.06$ \\
Nutrients (\% DM) & & & & \\
Crude protein & $46.5 \pm 0.47$ & $47.4 \pm 0.01$ & $43.0 \pm 0.04$ & $42.1 \pm 0.26$ \\
Crude lipids & $2.9 \pm 0.18$ & $5.7 \pm 0.17$ & $2.8 \pm 0.06$ & $4.1 \pm 0.02$ \\
Ash & $5.7 \pm 0.00$ & $5.9 \pm 0.01$ & $5.5 \pm 0.00$ & $5.5 \pm 0.00$ \\
Total phosphorus & $0.6 \pm 0.02$ & $0.6 \pm 0.01$ & $0.5 \pm 0.01$ & $0.4 \pm 0.02$ \\
Gross energy (MJ/kg DM) & $21.8 \pm 0.01$ & $22.32 \pm 0.02$ & $21.1 \pm 0.01$ & $21.5 \pm 0.01$ \\
Cell wall polysaccharides $(\boldsymbol{\%} \mathbf{D M})^{\mathbf{3}}$ & & & \\
$\beta$-glucan & $16.4 \pm 3.19$ & $11.1 \pm 0.84$ & $15 \pm 1.41$ & $11.8 \pm 0.73$ \\
Mannan & $7.9 \pm 2.16$ & $6.0 \pm 0.66$ & $11.3 \pm 0.95$ & $10.4 \pm 0.67$ \\
Chitin & $0.3 \pm 0.07$ & $0.2 \pm 0.02$ & $0.5 \pm 0.05$ & $0.4 \pm 0.08$ \\
\hline
\end{tabular}

${ }^{1} \mathrm{DM}$ - dry matter.

${ }^{2}$ Crude protein, crude lipids, ash, total phosphorus, and gross energy contents of yeasts are mean values \pm SD from duplicate analyses.

${ }^{3} \beta$-glucan, mannan and chitin contents of yeasts are mean values $\pm \mathrm{SD}$ from triplicate analyses. 


\section{Supplementary Note 2: Supplemental figures}

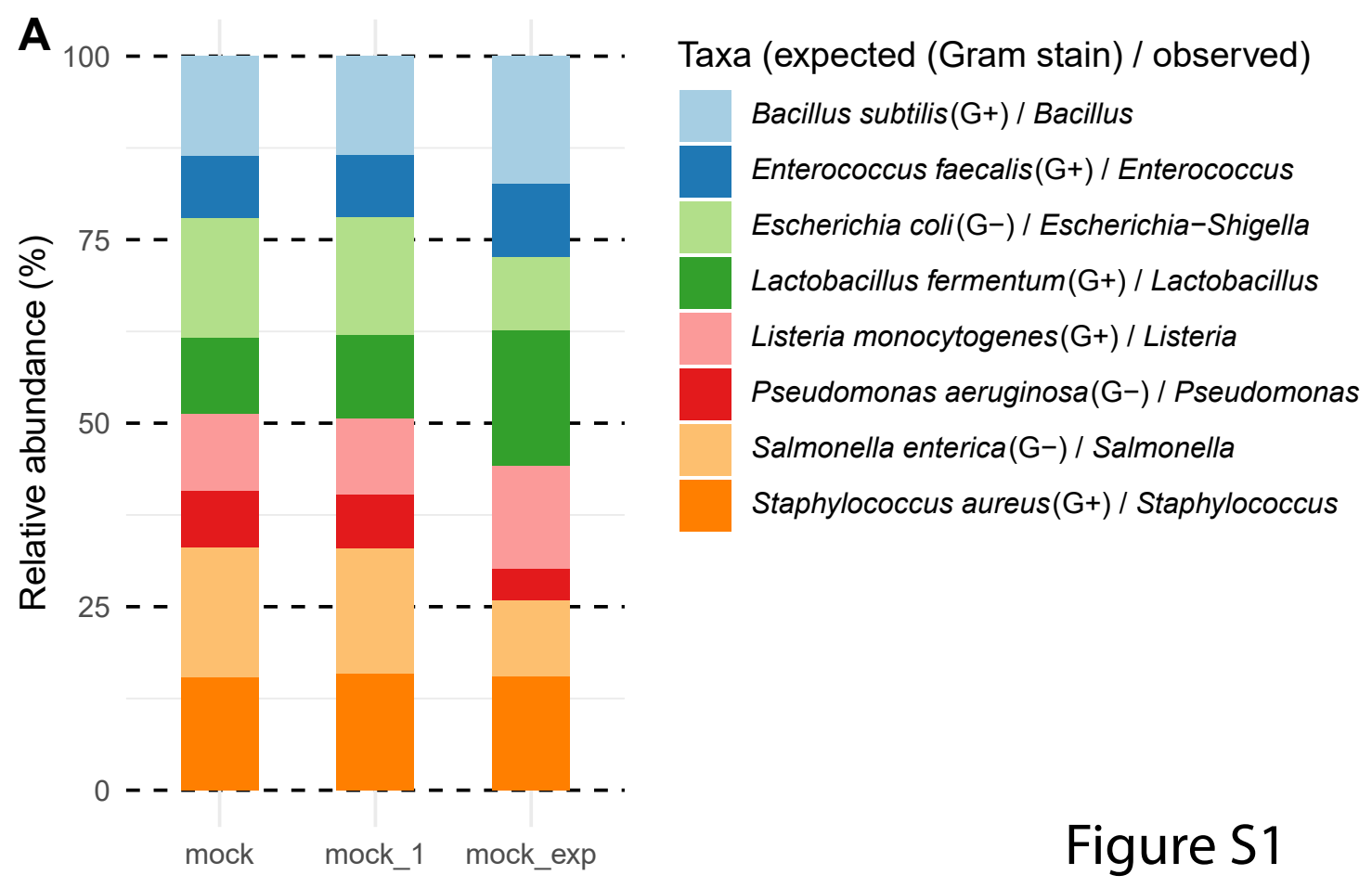

Fig. S1. Expected (mock_exp) and observed (mock and mock_1) taxonomic profiles of the mock microbial community standard). 

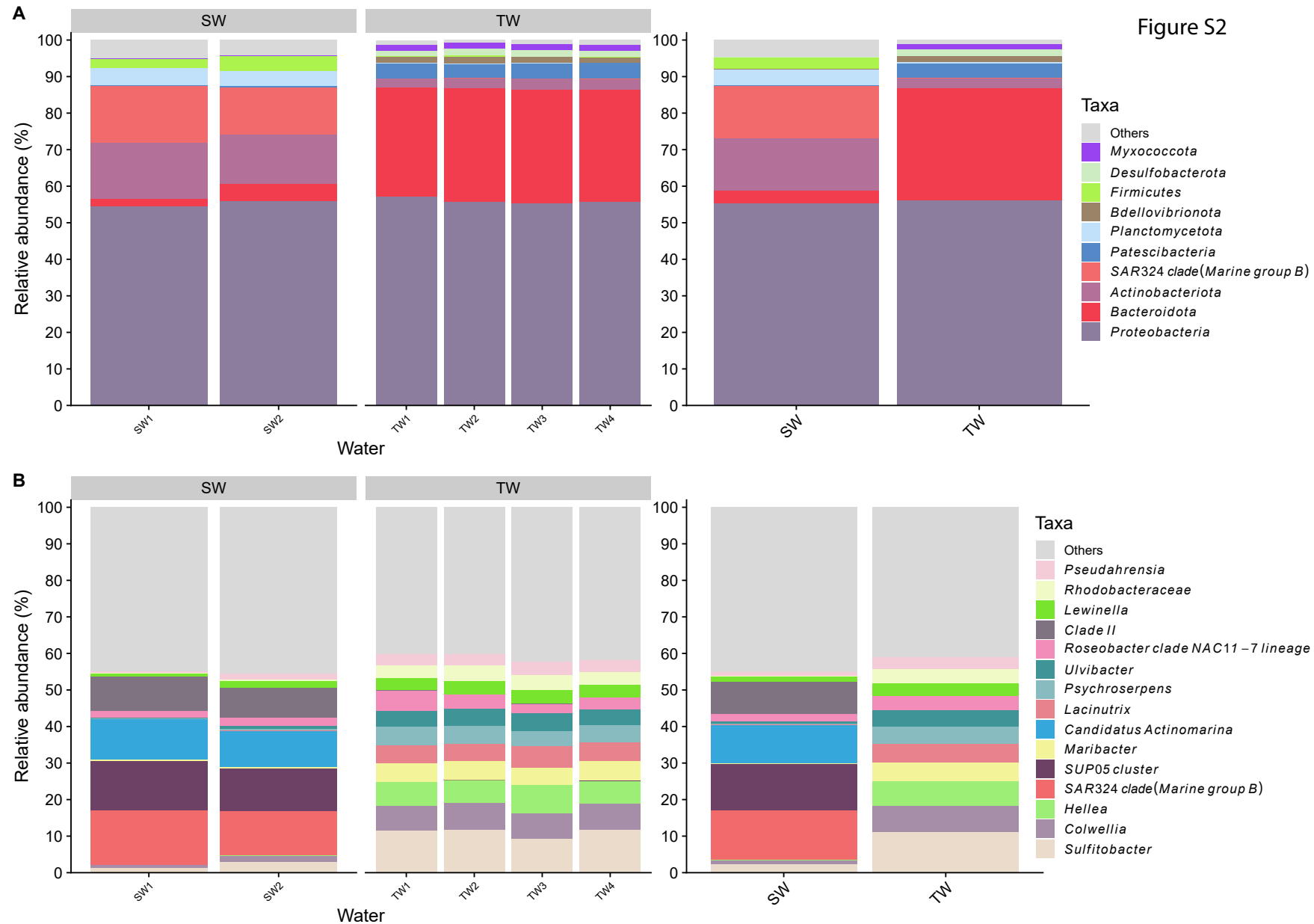

Fig. S2. Microbiota composition of water samples. Relative abundance of the top 10 most abundant taxa at phylum level (A) and top 15 most abundant taxa at genus or lowest taxonomic rank (B). The mean relative abundance of each taxon within the same water type is displayed on the right side. The samples are group water type; SW - water collected from the source tank (i.e., header tank) and TW - water collected from the fish rearing tanks. Water collected from the 18 fish tanks were mixed, four subsamples were taken and used for the microbiota analysis. 
$\mathrm{A}_{1}$

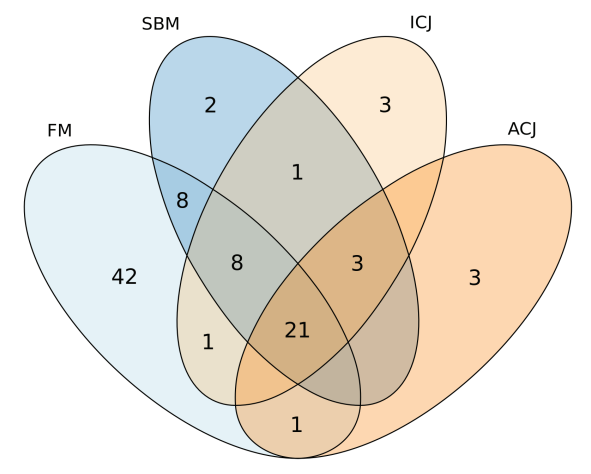

B 5

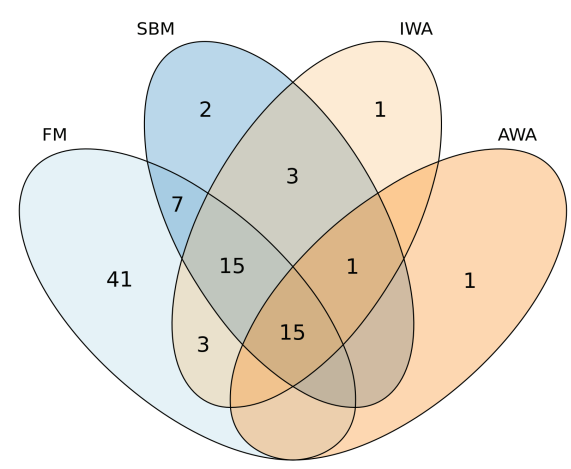

Fig. S3. Venn's diagram ( $A$ and B) showing the shared and the unique amplicon sequence variants (ASVs) in the digesta sample of fish fed the experimental diets. The ASVs were computed using a prevalence threshold of $80 \%$. FM - fishmeal-based; SBM - soybean meal-based; 4 experimental diets containing $300 \mathrm{~g} / \mathrm{kg} \mathrm{SBM}$ and $100 \mathrm{~g} / \mathrm{kg}$ of ICJ - inactivated Cyberlindnera jadinii; ACJ - autolyzed C. jadinii; IWA - inactivated Wickerhamomyces anomalus; AWA - autolyzed W. anomalus diets. 
A
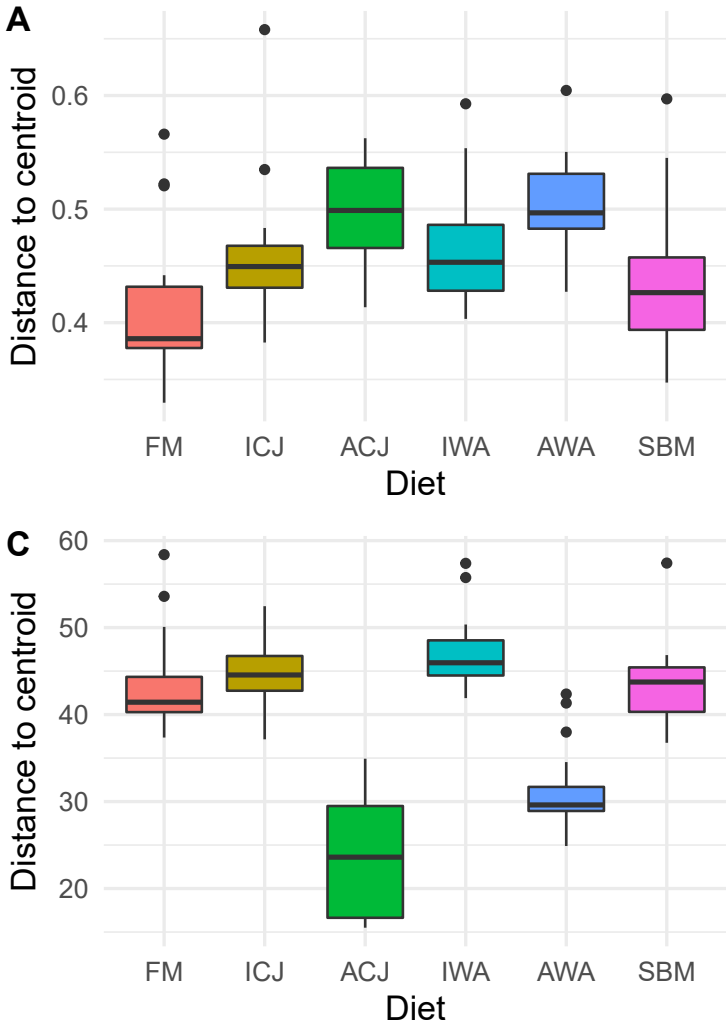

B

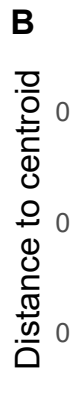

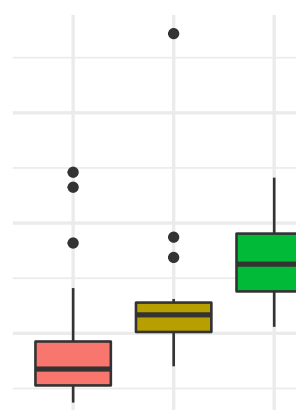

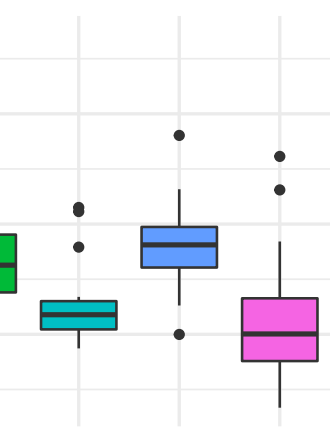

FM ICJ ACJ IWA AWA SBM Diet

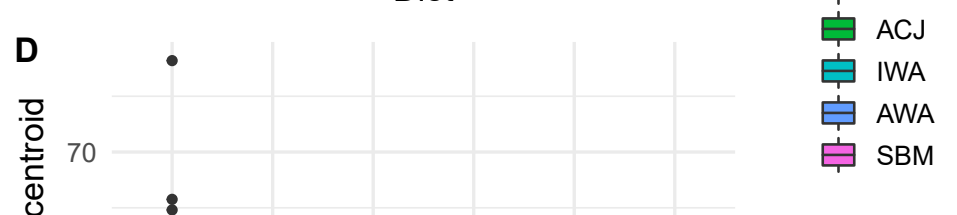

Figure S4

Diet

官 FM

官 ICJ

ACJ

官 IWA

AWA

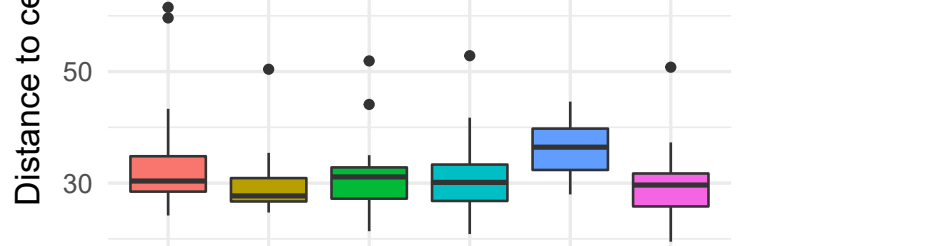

Fig. S4. Boxplots for homogeneity of multivariate dispersions (PERMDISP) in gut microbiota of fish fed experimental diets. The PERMDISP test was based on; (A) Jaccard distance, (B) Unweighted Unifrac distance (C) Aitchison distance and (D) PhILR transformed Euclidean distance. FM - fishmeal-based; SBM - soybean meal-based; 4 experimental diets containing $300 \mathrm{~g} / \mathrm{kg}$ SBM and $100 \mathrm{~g} / \mathrm{kg}$ of ICJ - inactivated Cyberlindnera jadinii; ACJ - autolyzed C. jadinii; IWA - inactivated Wickerhamomyces anomalus; AWA - autolyzed $W$. anomalus diets. 
a

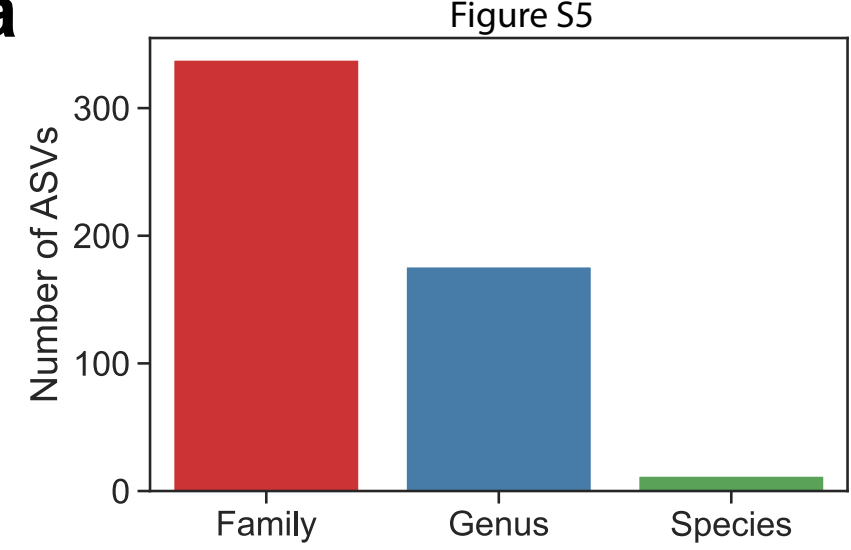

b

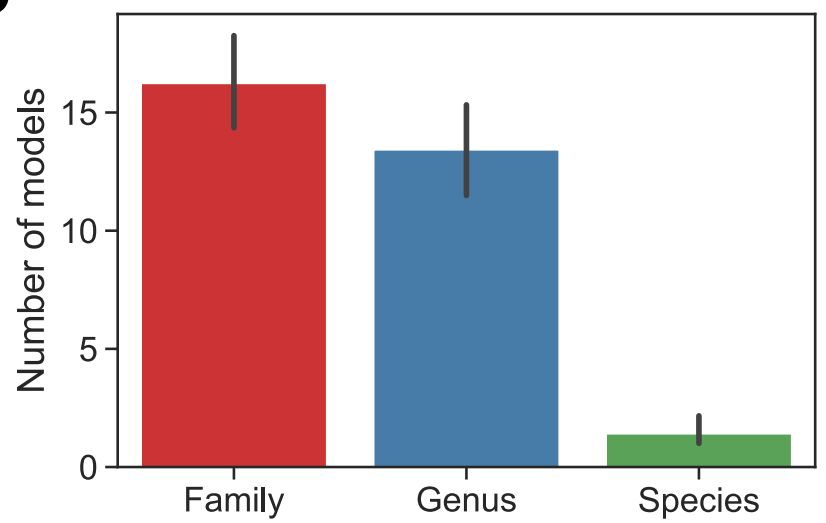

Fig. S5. Number of ASVs mapped to genome-scale metabolic models. Number of samples matched to models at different taxonomic levels $(\mathbf{A})$ and the number of models mapped to each sample by taxonomic level (B). 

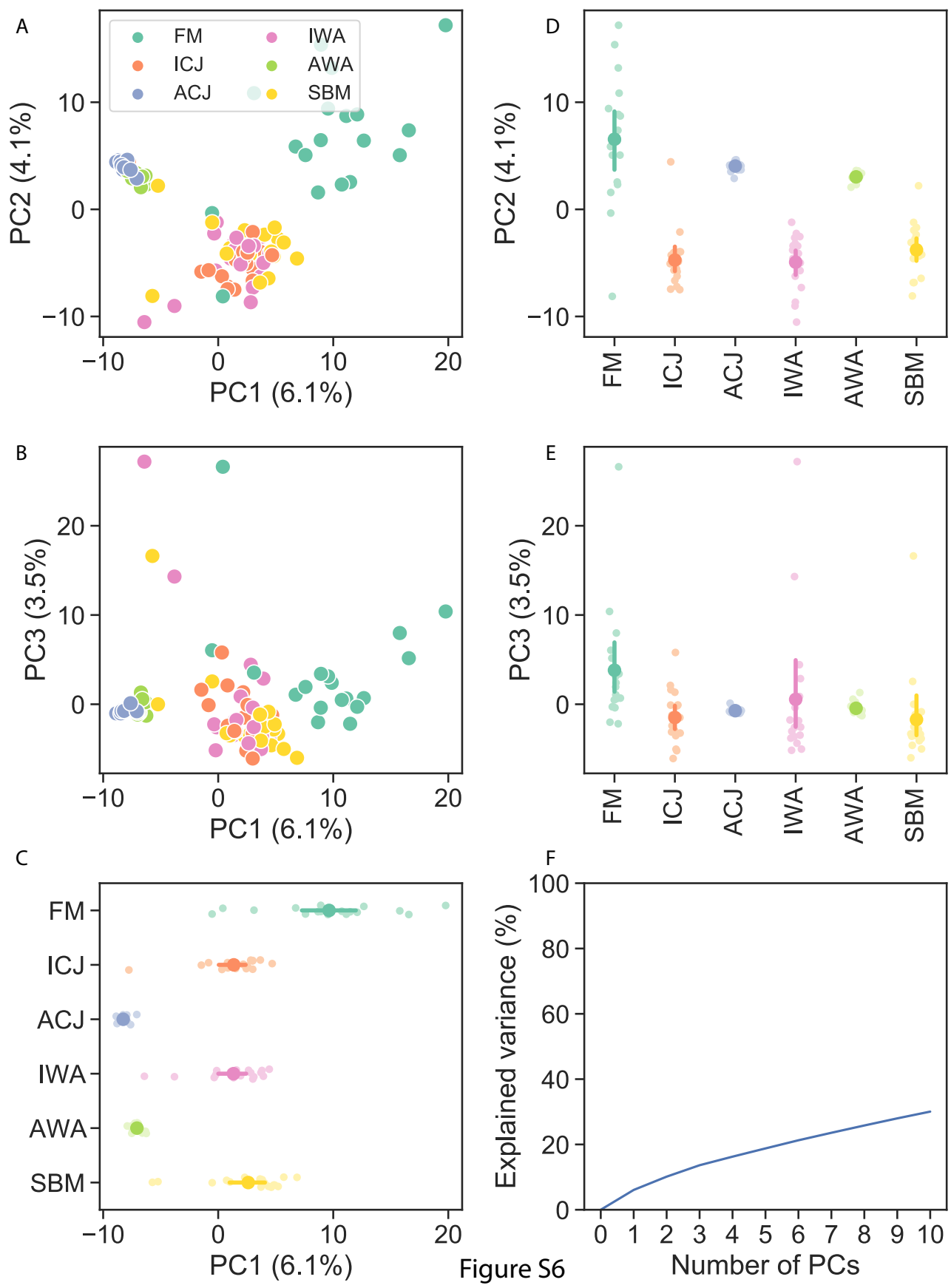

Fig. S6. Principal component (PC) analysis on standardized amplicon sequence variants (ASVs). Score plots for PC1 and PC2 (A) and PC1 and PC3 (B), mean scores with 95\% confidence intervals for PC1 (C), PC2 (d), and PC3 (E), and percentage of variance explained by PCs (F).FM - fishmeal-based; SBM - soybean meal-based; 4 experimental diets containing $300 \mathrm{~g} / \mathrm{kg}$ SBM and $100 \mathrm{~g} / \mathrm{kg}$ of ICJ - inactivated Cyberlindnera jadinii; ACJ - autolyzed C. jadinii; IWA - inactivated Wickerhamomyces anomalus; AWA autolyzed W. anomalus diets. 

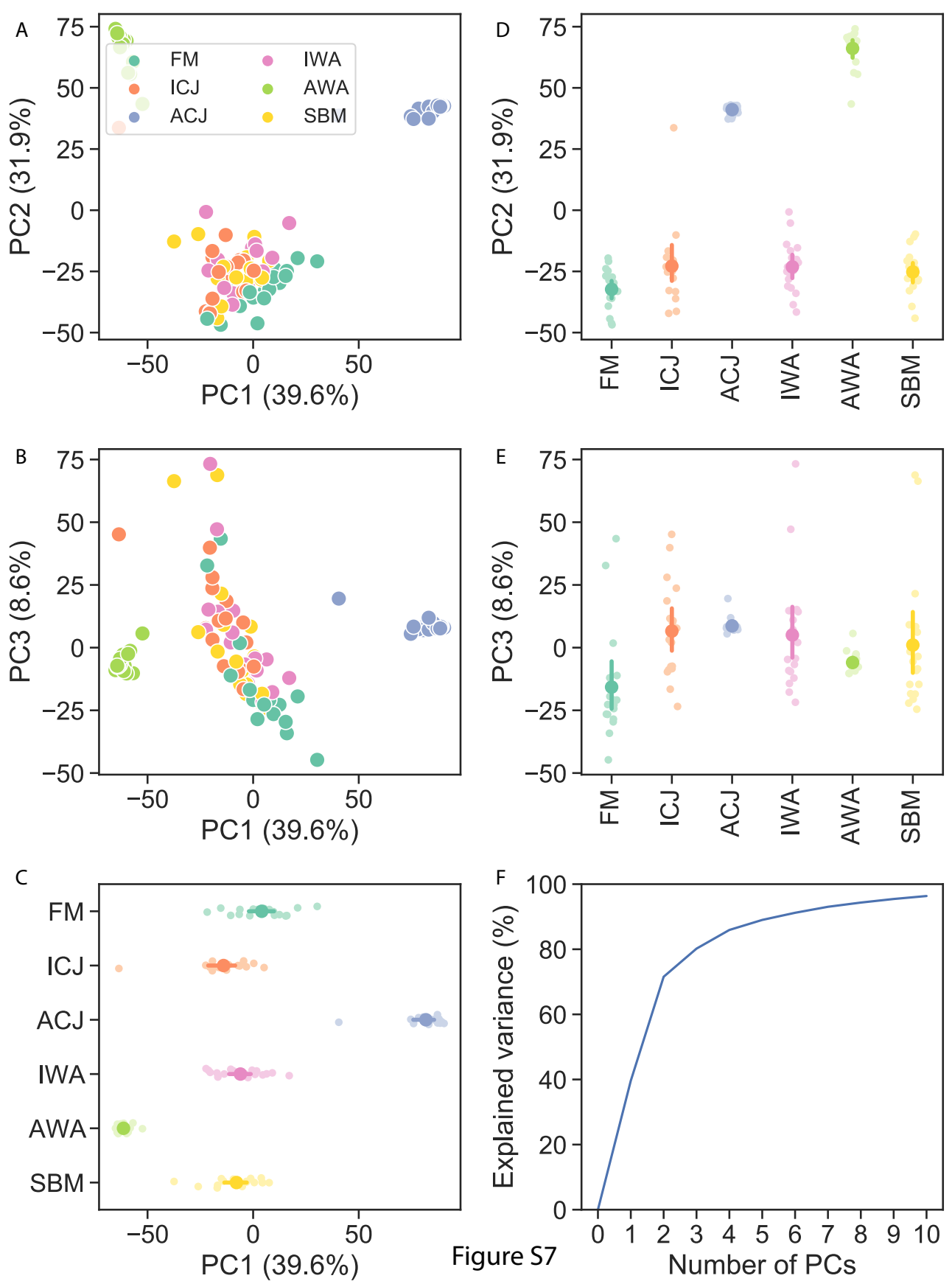

Fig. S7. Principal component (PC) analysis on standardized amplicon sequence variants (ASVs). Score plots for PC1 and PC2 (A) and PC1 and PC3 (B), mean scores with 95\% confidence intervals for PC1 (C), PC2 (d), and PC3 (E), and percentage of variance explained by PCs (F).FM - fishmeal-based; SBM - soybean meal-based; 4 experimental diets containing $300 \mathrm{~g} / \mathrm{kg} \mathrm{SBM} \mathrm{and} 100 \mathrm{~g} / \mathrm{kg}$ of ICJ - inactivated Cyberlindnera jadinii; ACJ - autolyzed C. jadinii; IWA - inactivated Wickerhamomyces anomalus; AWA autolyzed $W$. anomalus diets. 
a

Figure S8

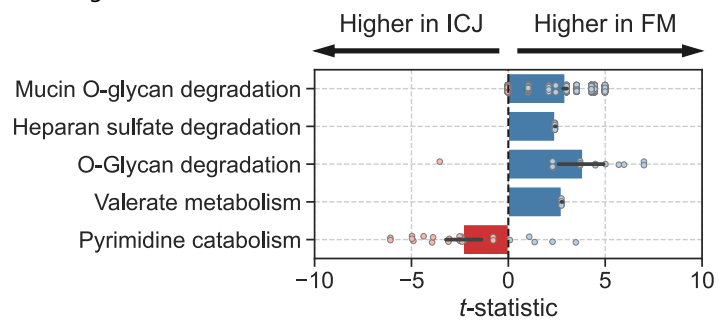

b

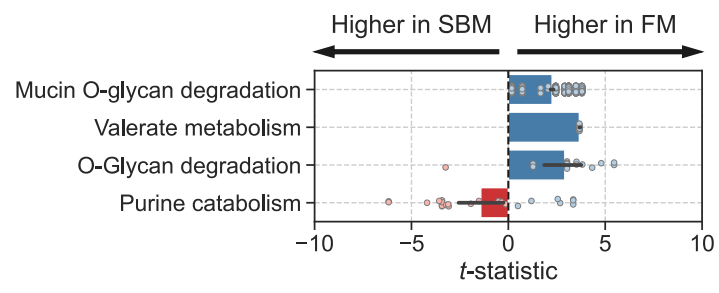

C

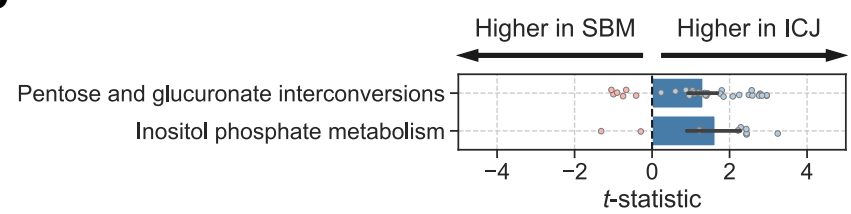

d

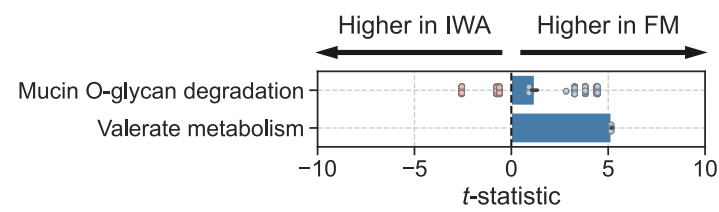

e

f

g
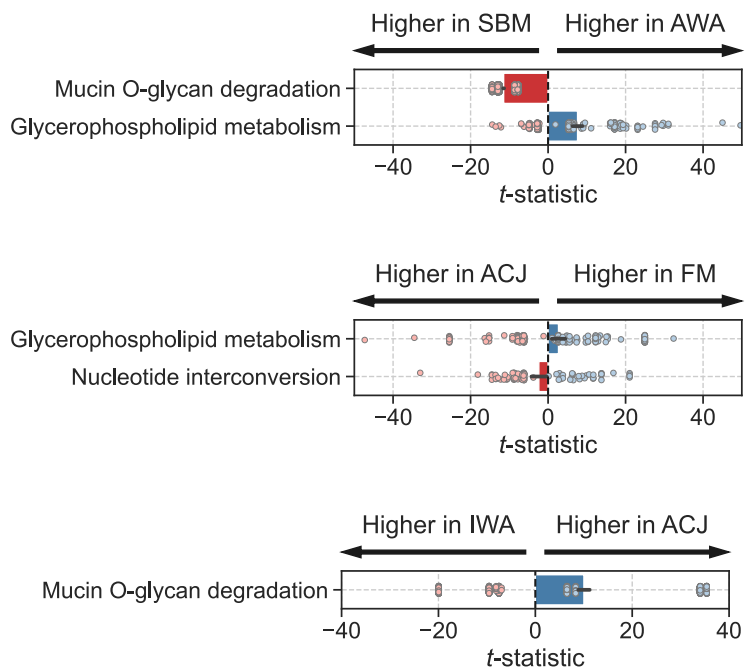

h

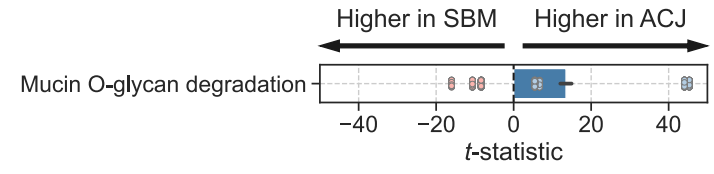

i

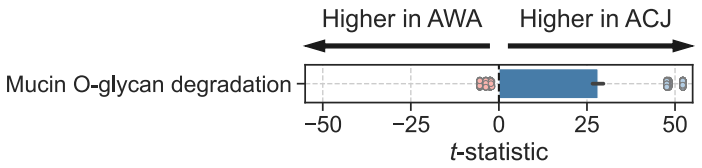

j

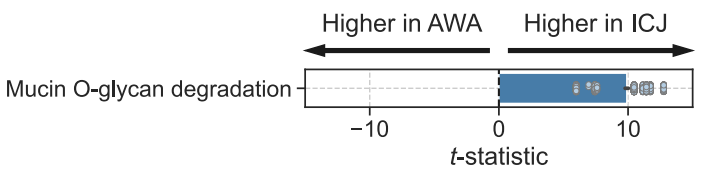

k
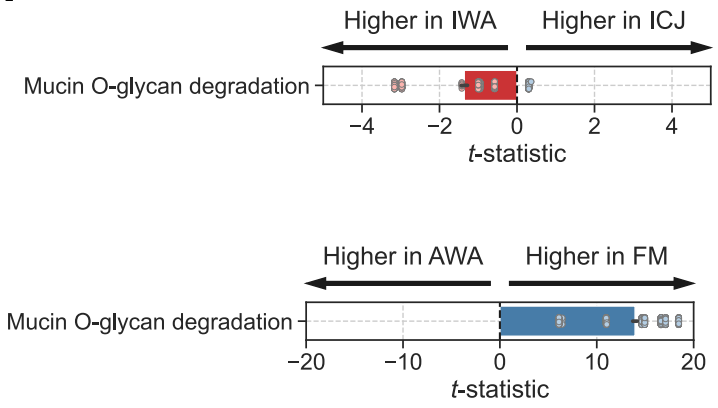

m

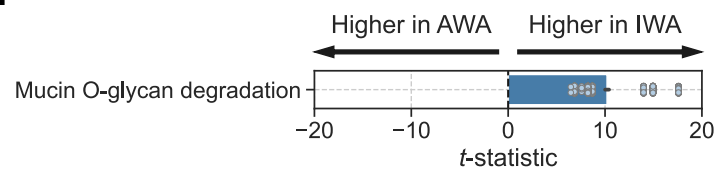

n

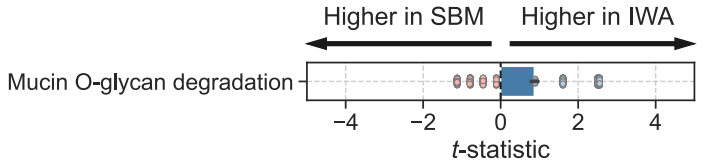

O

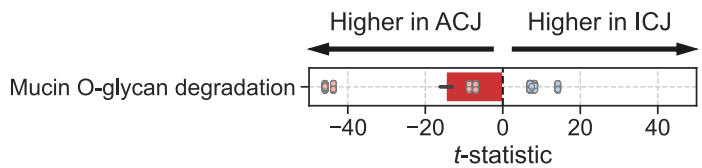

Fig. S8. The $t$-statistic tests comparing reaction abundances between each pair of diets. The $t$-statistic for each reaction is shown along with the mean across all reactions with $95 \%$ confidence interval for all significantly enriched subsystems. FM fishmeal-based; SBM - soybean meal-based; 4 experimental diets containing $300 \mathrm{~g} / \mathrm{kg} \mathrm{SBM}$ and $100 \mathrm{~g} / \mathrm{kg}$ of ICJ - inactivated Cyberlindnera jadinii; ACJ - autolyzed C. jadinii; IWA - inactivated Wickerhamomyces anomalus; AWA - autolyzed W. anomalus diets. 


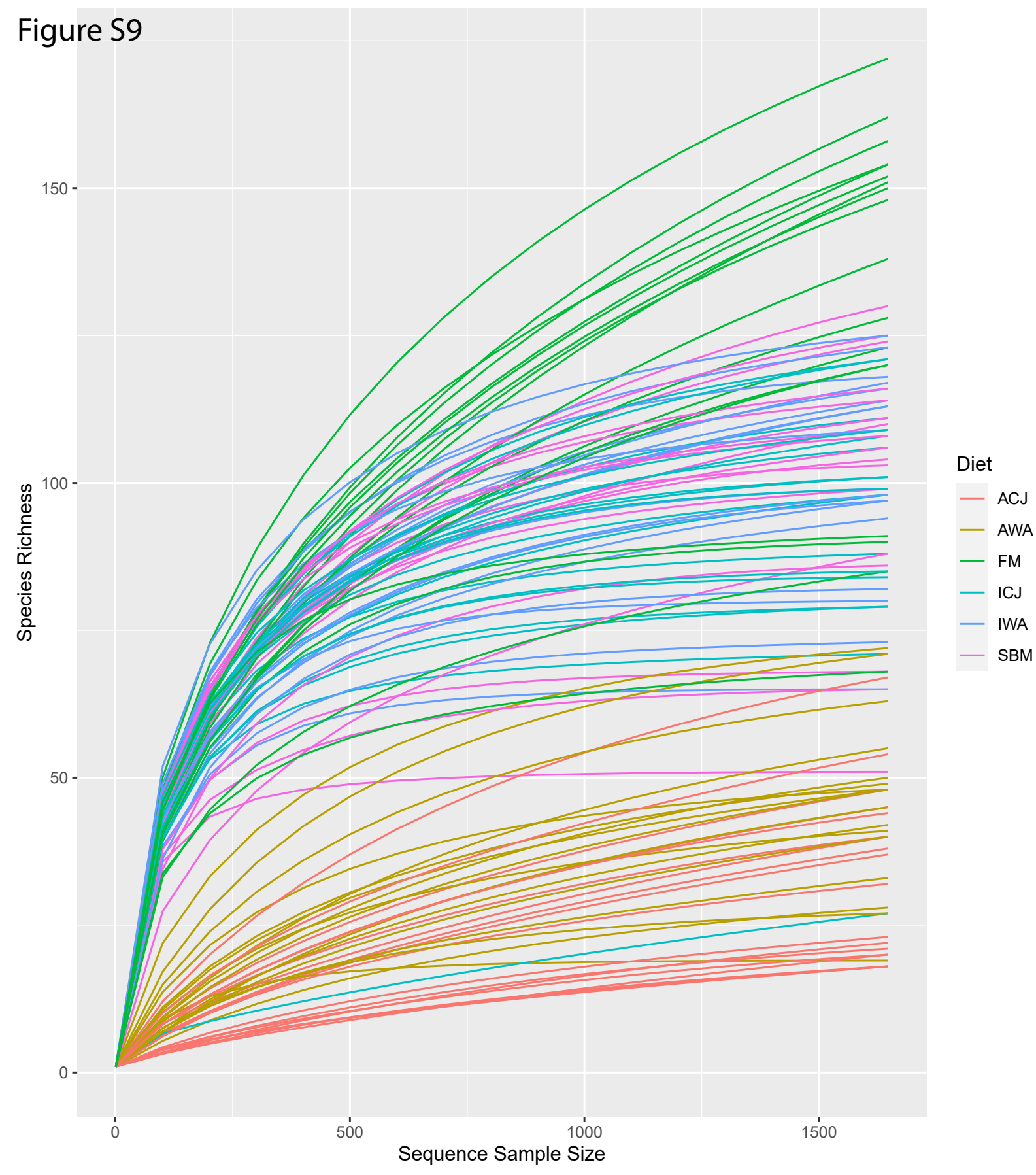

Fig. S9. Rarefaction curves showing subsampling of sample into minimum sample sequence (1,604 sequence per sample). The rarefied amplicon sequence variants table was used for computation of Jaccard and unweighted Unifrac beta-diversity distances. FM - fishmeal-based; SBM - soybean meal-based; 4 experimental diets containing $300 \mathrm{~g} / \mathrm{kg} \mathrm{SBM}$ and $100 \mathrm{~g} / \mathrm{kg}$ of ICJ - inactivated Cyberlindnera jadinii; ACJ - autolyzed C. jadinii; IWA - inactivated Wickerhamomyces anomalus; AWA - autolyzed W. anomalus diets. 


\section{Supplementary Files}

This is a list of supplementary files associated with this preprint. Click to download.

- Additionalfile2supplementarytablesTablesS1andS2.xlsx 